\title{
Palladium-Catalyzed Intramolecular Dearomatization of Indoles via Decarboxylative Alkynyl Termination
}

Si Chen, Xin-Xing Wu, Jia Wang, Xin-Hua Hao, Yu Xia, Yi Shen, Huanwang Jing* and Yong-Min Liang*

State Key Laboratory of Applied Organic Chemistry, Lanzhou University, Lanzhou, 730000, P.R. China.

Email: liangym@1zu.edu.cn; hwjing@1zu.edu.cn.

\section{Supporting Information}

\section{Content:}

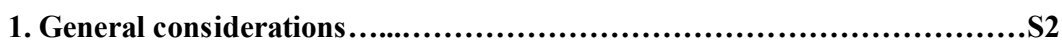

2. Optimization of the reaction conditions ....................................S2

3. Preparation of starting materials .........................................S2

4. Experiment procedure.............................................S2-S3

5. Spectra Data........................................................S4-S11

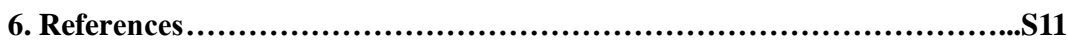

7. Crystallographic data of 3a................................................S12

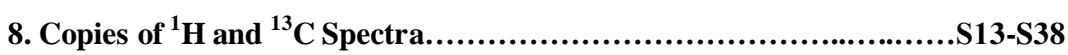




\section{General considerations.}

Unless stated otherwise, all reactions were carried out in flame-dried glassware under a dry argon atmosphere. All solvents were purified and dried according to standard methods prior to use. For product purification by flash column chromatography, silica gel (200 300 mesh) and light petroleum ether (bp. 60 90) are used. ${ }^{1} \mathrm{H}$ NMR spectra were recorded on a Bruker advance III $400 \mathrm{MHz}$ in $\mathrm{CDCl}_{3}$ and ${ }^{13} \mathrm{C}$ NMR spectra were recorded on 100 $\mathrm{MHz}$ in $\mathrm{CDCl}_{3}$ using TMS as internal standard. Data for ${ }^{1} \mathrm{H}$ NMR are recorded as follows: chemical shift $(\delta, \mathrm{ppm})$, multiplicity ( $\mathrm{s}=$ singlet, $\mathrm{d}=$ doublet, $\mathrm{t}=$ triplet, $\mathrm{m}=$ multiplet or unresolved, $\mathrm{br}=$ broad singlet, coupling constant (s) in $\mathrm{Hz}$, integration). Data for ${ }^{13} \mathrm{C}$ NMR is reported in terms of chemical shift $(\delta$, ppm). IR spectra were obtained on a Perkin-Elmer Model 2000 FT-IR using KBr plates (thin film). High-resolution mass spectral analysis (HRMS) data were measured on a Bruker Daltonics Apex II 47e Specifications.

\section{Optimization of the reaction conditions.}<smiles>Cc1cc2ccccc2n1C(=O)c1ccccc1Br</smiles>

$1 \mathrm{a}$

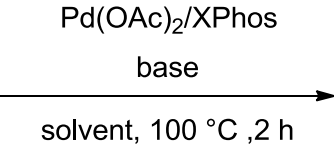

2a

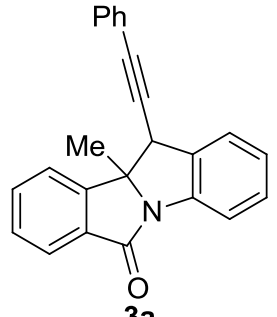

3a

\begin{tabular}{|l|l|l|l|l|}
\hline entry & base & solvent & yield $^{a}(\%)$ & $\mathrm{dr}^{b}$ \\
\hline 1 & $\mathrm{~K}_{2} \mathrm{CO}_{3}$ & toluene & 25 & $18: 1$ \\
\hline 2 & $t$-BuOLi & toluene & trace & - \\
\hline 3 & $\mathrm{~K}_{3} \mathrm{PO}_{4}$ & toluene & 60 & $16: 1$ \\
\hline 3 & $t$-BuOK & toluene & 45 & $17: 1$ \\
\hline 4 & $\mathrm{DBU}$ & toluene & 0 & - \\
\hline 5 & $\mathrm{Et}_{3} \mathrm{~K}$ & toluene & trace & - \\
\hline 6 & $\mathrm{KOAc}$ & toluene & 57 & $19: 1$ \\
\hline 7 & $\mathrm{Cs}_{2} \mathrm{CO}_{3}$ & MeCN & 0 & - \\
\hline 8 & $\mathrm{Cs}_{2} \mathrm{CO}_{3}$ & dioxane & 48 & $17: 1$ \\
\hline 9 & $\mathrm{Cs}_{2} \mathrm{CO}_{3}$ & DMF & 0 & - \\
\hline 10 & $\mathrm{Cs}_{2} \mathrm{CO}_{3}$ & DCE & 57 & $16: 1$ \\
\hline 11 & $\mathrm{Cs}_{2} \mathrm{CO}_{3}$ & DMSO & 0 & - \\
\hline 12 & $\mathrm{Cs}_{2} \mathrm{CO}_{3}$ & $i$ PrOH & 0 & - \\
\hline
\end{tabular}

${ }^{a}$ Reaction conditions unless otherwise noted: $1(0.2 \mathrm{mmol}), 2(0.3 \mathrm{mmol}), \operatorname{Pd}(\mathrm{MeCN})_{2} \mathrm{Cl}_{2}(5 \mathrm{~mol} \%), \mathrm{XPhos}(10$ mol \%), base (2.0 equiv), toluene $(2.0 \mathrm{~mL}), 100{ }^{\circ} \mathrm{C}, 2 \mathrm{~h}$. Isolated yields are shown. ${ }^{b} \mathrm{The} \mathrm{dr}$ values were determined by ${ }^{1} \mathrm{H}$ NMR analysis of the crude reaction mixture.

\section{Preparation of starting materials.}

Compounds 1 were prepared according to the known procedures ${ }^{1,2}$. Compouds $\mathbf{2}$ were prepared according to the known procedures ${ }^{3}$. All other starting materials were commercially available.

\section{General procedure for the preparation of the product 3.}

$1(0.2 \mathrm{mmol}), 2(0.3 \mathrm{mmol}), \mathrm{Pd}(\mathrm{MeCN})_{2} \mathrm{Cl}_{2}(5 \mathrm{~mol} \%), \mathrm{XPhos}(10 \mathrm{~mol} \%), \mathrm{Cs}_{2} \mathrm{CO}_{3}(2.0$ equiv) were added to a sealed tube, toluene $(2.0 \mathrm{~mL})$ was added via syringe. The mixture was flushed with Ar and stirred at $100{ }^{\circ} \mathrm{C}$ until 
completion (monitored by TLC). After cooling at room temperature, the mixture was diluted with diethyl ether, washed with water, dried over magnesium sulfate and purified by flash chromatography on silica gel (Hex/EA: 20/1-10/1). 


\section{Spectra Data.}

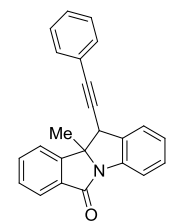

(10bS, 11R)-10b-methyl-11-(phenylethynyl)-10b,11-dihydro-6H-isoindolo[2,1-a]indol-6-one (3a): yellow solid (87\%, $58.3 \mathrm{mg})$; m.p. $152-154{ }^{\circ} \mathrm{C} ;{ }^{1} \mathrm{H} \mathrm{NMR}\left(\mathrm{CDCl}_{3}, 400 \mathrm{MHz}\right) \delta: 7.91(\mathrm{~d}, J=7.2 \mathrm{~Hz}, 1 \mathrm{H}), 7.73(\mathrm{~d}, J=8.0 \mathrm{~Hz}, 1 \mathrm{H})$, $7.66(\mathrm{t}, J=7.2 \mathrm{~Hz}, 1 \mathrm{H}), 7.60(\mathrm{~d}, J=7.2 \mathrm{~Hz}, 1 \mathrm{H}), 7.54(\mathrm{t}, J=7.2 \mathrm{~Hz}, 1 \mathrm{H}), 7.46(\mathrm{t}, J=7.2 \mathrm{~Hz}, 1 \mathrm{H}), 7.40(\mathrm{t}, J=7.6$ $\mathrm{Hz} 1 \mathrm{H}), 7.12-7.20(\mathrm{~m}, 2 \mathrm{H}), 7.08(\mathrm{t}, J=7.2 \mathrm{~Hz}, 2 \mathrm{H}), 7.70(\mathrm{~d}, J=7.2 \mathrm{~Hz}, 2 \mathrm{H}), 4.23(\mathrm{~s}, 1 \mathrm{H}), 1.71(\mathrm{~s}, 1 \mathrm{H}) .{ }^{13} \mathrm{C} \mathrm{NMR}$ $\left(100 \mathrm{MHz}, \mathrm{CDCl}_{3}\right) \delta: 168.6,149.0,138.7,135.6,132.8,132.5,131.1,129.1,128.8,127.9,127.9,126.0,125.0$, 124.6, 123.1, 122.4, 117.6, 86.9, 86.6, 75.2, 43.1, 26.7. IR: 3397, 2924, 1706, 1602, 1478, 1355, 1307, 1243, 1140, 755, 693. HRMS-ESI (m/z) $[\mathrm{M}+\mathrm{H}]^{+}$calcd for $\mathrm{C}_{24} \mathrm{H}_{18} \mathrm{NO}$ : 336.1383; Found, 336.1390.

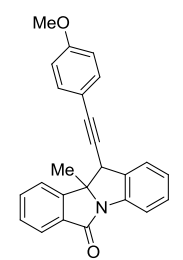

(10bS, 11R)-11-((4-methoxyphenyl)ethynyl)-10b-methyl-10b,11-dihydro-6H-isoindolo[2,1-a]indol-6-one (3b): yellow solid (84\%, $61.3 \mathrm{mg})$; m.p. 196-198 ${ }^{\circ} \mathrm{C} ;{ }^{1} \mathrm{H} \mathrm{NMR}\left(\mathrm{CDCl}_{3}, 400 \mathrm{MHz}\right) \delta: 7.89$ (d, $\left.J=7.6 \mathrm{~Hz}, 1 \mathrm{H}\right), 7.73(\mathrm{~d}, J$ $=7.6 \mathrm{~Hz}, 1 \mathrm{H}), 7.63(\mathrm{t}, J=7.2 \mathrm{~Hz}, 1 \mathrm{H}), 7.57(\mathrm{~d}, J=7.2 \mathrm{~Hz}, 1 \mathrm{H}), 7.51(\mathrm{t}, J=7.2 \mathrm{~Hz}, 1 \mathrm{H}), 7.44(\mathrm{~d}, J=7.2 \mathrm{~Hz}, 1 \mathrm{H})$, $7.37(\mathrm{t}, J=7.6 \mathrm{~Hz}, 1 \mathrm{H}), 7.16(\mathrm{t}, J=7.2 \mathrm{~Hz}, 1 \mathrm{H}), 6.70(\mathrm{~d}, J=7.6 \mathrm{~Hz}, 2 \mathrm{H}), 6.60(\mathrm{~d}, J=7.6 \mathrm{~Hz}, 2 \mathrm{H}), 4.20(\mathrm{~s}, 1 \mathrm{H})$, $3.68(\mathrm{~s}, 3 \mathrm{H}), 1.69(\mathrm{~s}, 3 \mathrm{H}) .{ }^{13} \mathrm{C} \mathrm{NMR}\left(100 \mathrm{MHz}, \mathrm{CDCl}_{3}\right) \delta: 168.6,159.2,149.1,138.7,135.9,132.8,132.5,132.4$, 129.0, 128.7, 126.0, 124.9, 124.5, 123.1, 117.6, 114.6, 113.5, 86.8, 85.2, 75,3, 55.1, 43.2, 26.6. IR: 3370, 2925, $1702,1604,1510,1478,1355,1289,1247,1173,1032,833,757,702$. HRMS-ESI $(\mathrm{m} / \mathrm{z})[\mathrm{M}+\mathrm{H}]^{+}$calcd for $\mathrm{C}_{25} \mathrm{H}_{20} \mathrm{NO}_{2}: 366.1489$; Found, 366.1487.

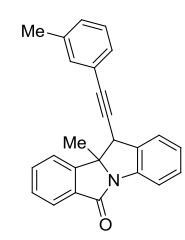

(10bS, 11R)-10b-methyl-11-(m-tolylethynyl)-10b,11-dihydro-6H-isoindolo[2,1-a]indol-6-one (3c): brown solid (87\%, $60.7 \mathrm{mg})$; m.p. 58-60 ${ }^{\circ} \mathrm{C} ;{ }^{1} \mathrm{H} \mathrm{NMR}\left(\mathrm{CDCl}_{3}, 400 \mathrm{MHz}\right) \delta: 7.90(\mathrm{~d}, J=7.2 \mathrm{~Hz}, 1 \mathrm{H}), 7.73(\mathrm{~d}, J=7.6 \mathrm{~Hz}, 1 \mathrm{H})$, $7.64(\mathrm{t}, J=7.2 \mathrm{~Hz}, 1 \mathrm{H}), 7.58(\mathrm{~d}, J=7.2 \mathrm{~Hz}, 1 \mathrm{H}), 7.52(\mathrm{t}, J=7.2 \mathrm{~Hz}, 1 \mathrm{H}), 7.44(\mathrm{~d}, J=7.2 \mathrm{~Hz}, 1 \mathrm{H}), 7.38(\mathrm{t}, J=7.6$ $\mathrm{Hz}, 1 \mathrm{H}), 7.16(\mathrm{t}, J=7.6 \mathrm{~Hz}, 1 \mathrm{H}), 6.94(\mathrm{~s}, 2 \mathrm{H}), 6.58(\mathrm{~s}, 2 \mathrm{H}), 4.21(\mathrm{~s}, 1 \mathrm{H}), 2.14(\mathrm{~s}, 3 \mathrm{H}), 1.69(\mathrm{~s}, 3 \mathrm{H}) .{ }^{13} \mathrm{C}$ NMR $(100$ $\left.\mathrm{MHz}, \mathrm{CDCl}_{3}\right) \delta: 168.6,149.1,138.7,137.5,135.7,132.8,132.5,131.8,129.0,128.8,128.7,128.1,127.7,126.0$, 124.9, 124.5, 123.1, 122.2, 117.6, 87.1, 86.3, 75.3, 43.1, 26.6, 21.0. IR: 3403, 2924, 1709, 1603, 1478, 1355, 1308, 1243, 1140, 1046, 756, 692. HRMS-ESI (m/z) [M + H] $]^{+}$calcd for $\mathrm{C}_{25} \mathrm{H}_{20} \mathrm{NO}$ : 350.1539; Found, 350.1538. 


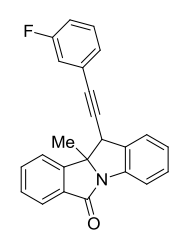

(10Bs，11R)-11-((3-fluorophenyl)ethynyl)-10b-methyl-10b,11-dihydro-6H-isoindolo[2,1-a]indol-6-one (3d): yellow solid (71\%, $50.1 \mathrm{mg})$; m.p. 120-122 ${ }^{\circ} \mathrm{C} ;{ }^{1} \mathrm{H}$ NMR $\left(\mathrm{CDCl}_{3}, 400 \mathrm{MHz}\right) \delta: 7.91(\mathrm{~d}, J=7.6 \mathrm{~Hz}, 1 \mathrm{H}), 7.73(\mathrm{~d}, J$ $=8.0 \mathrm{~Hz}, 1 \mathrm{H}), 7.66(\mathrm{t}, J=7.6 \mathrm{~Hz}, 1 \mathrm{H}), 7.52-7.59(\mathrm{~m}, 2 \mathrm{H}), 7.44(\mathrm{~d}, J=7.6 \mathrm{~Hz}, 1 \mathrm{H}), 7.39(\mathrm{t}, J=7.6 \mathrm{~Hz}, 1 \mathrm{H}), 7.17(\mathrm{t}$, $J=7.6 \mathrm{~Hz}, 1 \mathrm{H}), 7.00-7.05(\mathrm{~m}, 1 \mathrm{H}), 6.81-6.86(\mathrm{~m}, 1 \mathrm{H}), 6.54(\mathrm{~d}, J=7.6 \mathrm{~Hz}, 1 \mathrm{H}), 6.44(\mathrm{~d}, J=9.2 \mathrm{~Hz}, 1 \mathrm{H}), 4.21(\mathrm{~s}$, 1H), $1.70(\mathrm{~s}, 3 \mathrm{H}) .{ }^{13} \mathrm{C}$ NMR (100 MHz, $\left.\mathrm{CDCl}_{3}\right) \delta: 168.5,163.2,160.7,148.9,138.7,135.3,132.8,132.5,129.5$, $129.4,129.2,128.9,127.0,127.0,126.0,125.0,124.6,124.3,124.2,123.1,118.1,117.8,117.7,115.4,115.2,87.6$, 85.8, 85.8, 75.2, 43.1, 26.6. IR: 3401, 3070, 2925, 1707, 1605, 1478, 1355, 1309, 1151, 1081, 872, 756, 682. HRMS-ESI (m/z) [M + H] $]^{+}$calcd for $\mathrm{C}_{24} \mathrm{H}_{17} \mathrm{FNO}$ : 354.1289; Found, 354.1287.

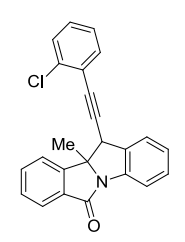

(10bS，11R)-11-((2-chlorophenyl)ethynyl)-10b-methyl-10b,11-dihydro-6H-isoindolo[2,1-a]indol-6-one (3e): brown solid $(85 \%, 62.7 \mathrm{mg})$; m.p. $124-126{ }^{\circ} \mathrm{C} ;{ }^{1} \mathrm{H} \mathrm{NMR}\left(\mathrm{CDCl}_{3}, 400 \mathrm{MHz}\right) \delta: 7.88(\mathrm{~d}, J=7.2 \mathrm{~Hz}, 1 \mathrm{H}), 7.74(\mathrm{~d}, J=$ $7.2 \mathrm{~Hz}, 1 \mathrm{H}), 7.61(\mathrm{~d}, J=8.8 \mathrm{~Hz}, 2 \mathrm{H}), 7.46-7.51(\mathrm{~m}, 2 \mathrm{H}), 7.38(\mathrm{t}, J=7.2 \mathrm{~Hz}, 1 \mathrm{H}), 7.17(\mathrm{t}, J=8.8 \mathrm{~Hz}, 2 \mathrm{H}), 7.04(\mathrm{t}$, $J=7.2 \mathrm{~Hz}, 1 \mathrm{H}), 6.95(\mathrm{t}, J=7.2 \mathrm{~Hz}, 1 \mathrm{H}), 6.76(\mathrm{~d}, J=7.2 \mathrm{~Hz}, 1 \mathrm{H}), 4.30(\mathrm{~s}, 1 \mathrm{H}), 1.70(\mathrm{~s}, 3 \mathrm{H}) .{ }^{13} \mathrm{C} \mathrm{NMR}(100 \mathrm{MHz}$, $\left.\mathrm{CDCl}_{3}\right) \delta: 168.4,148.8,138.6,135.5,135.3,133.0,132.8,132.6,129.1,128.9,128.8,128.8,126.0,126.0,125.0$, 124.6, 123.3, 122.3, 117.6, 91.6, 83.7, 75.0,43.2, 26.9. IR: 3394, 2923, 1704, 1602, 1476, 1356, 1307, 1130, 1033, 753, 698. HRMS-ESI (m/z) [M + H] $]^{+}$calcd for $\mathrm{C}_{24} \mathrm{H}_{17} \mathrm{ClNO} 370.0993$; Found, 370.0994.

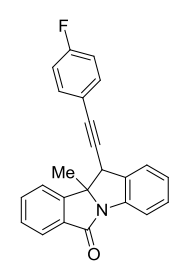

(10bS, 11R)-11-((4-fluorophenyl)ethynyl)-10b-methyl-10b,11-dihydro-6H-isoindolo[2,1-a]indol-6-one (3f): brown solid (90\%, $63.5 \mathrm{mg})$; m.p. 90-92 ${ }^{\circ} \mathrm{C} ;{ }^{1} \mathrm{H} \mathrm{NMR}\left(\mathrm{CDCl}_{3}, 400 \mathrm{MHz}\right) \delta: 7.90(\mathrm{~d}, J=7.6 \mathrm{~Hz}, 1 \mathrm{H}), 7.73(\mathrm{~d}, J=$ $7.6 \mathrm{~Hz}, 1 \mathrm{H}), 7.64(\mathrm{t}, J=7.2 \mathrm{~Hz}, 1 \mathrm{H}), 7.58(\mathrm{~d}, J=7.2 \mathrm{~Hz}, 1 \mathrm{H}), 7.52(\mathrm{t}, J=7.6 \mathrm{~Hz}, 1 \mathrm{H}), 7.44(\mathrm{~d}, J=7.2 \mathrm{~Hz}, 1 \mathrm{H})$, $7.38(\mathrm{t}, J=7.6 \mathrm{~Hz}, 1 \mathrm{H}), 7.17(\mathrm{t}, J=7.2 \mathrm{~Hz}, 1 \mathrm{H}), 6.74(\mathrm{~s}, 4 \mathrm{H}), 4.20(\mathrm{~s}, 1 \mathrm{H}), 1.70(\mathrm{~s}, 3 \mathrm{H}) .{ }^{13} \mathrm{C} \mathrm{NMR}(100 \mathrm{MHz}$, $\left.\mathrm{CDCl}_{3}\right) \delta: 168.5,163.4,160.9,149.0,138.7,135.6,133.0,132.9,132.8,132.4,129.1,128.8,126.0,125.0,124.6$, 123.1, 118.5, 118.4, 117.7, 115.2, 115.0, 86.3, 85.9, 75.3, 43.1, 26.5. IR: 3394, 2924, 1706, 1601, 1507, 1478, 1354, 1308, 1227, 1140, 837, 756, 703. HRMS-ESI (m/z) [M + H] calcd for $\mathrm{C}_{24} \mathrm{H}_{17}$ FNO: 354.1289; Found, 354.1292. 


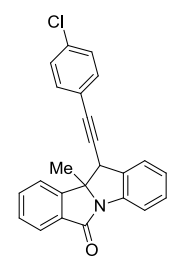

(10bS，11R)-11-((4-chlorophenyl)ethynyl)-10b-methyl-10b,11-dihydro-6H-isoindolo[2,1-a]indol-6-one (3g): brown solid (69\%, $50.9 \mathrm{mg})$; m.p. $84-86{ }^{\circ} \mathrm{C} ;{ }^{1} \mathrm{H} \mathrm{NMR}\left(\mathrm{CDCl}_{3}, 400 \mathrm{MHz}\right) \delta: 7.90(\mathrm{~d}, J=7.6 \mathrm{~Hz}, 1 \mathrm{H}), 7.73(\mathrm{~d}, J=$ $8.0 \mathrm{~Hz}, 1 \mathrm{H}), 7.62-7.66(\mathrm{~m}, 1 \mathrm{H}), 7.56(\mathrm{t}, J=7.6 \mathrm{~Hz}, 1 \mathrm{H}), 7.50-7.54(\mathrm{~m}, 1 \mathrm{H}), 7.44(\mathrm{~d}, J=7.6 \mathrm{~Hz}, 1 \mathrm{H}), 7.37-7.41(\mathrm{~m}$, 1H), 7.15-7.19 (m, 1H), $7.05(\mathrm{~d}, J=7.6 \mathrm{~Hz}, 2 \mathrm{H}), 6.68(\mathrm{~d}, J=7.2 \mathrm{~Hz}, 2 \mathrm{H}), 4.20(\mathrm{~s}, 1 \mathrm{H}), 1.70(\mathrm{~s}, 3 \mathrm{H}) .{ }^{13} \mathrm{C}$ NMR $\left(100 \mathrm{MHz}, \mathrm{CDCl}_{3}\right) \delta: 168.5,148.9,138.7,135.4,133.9,132.8,132.5,132.3,129.2,128.8,128.2,126.0,125.0$, 124.6, 123.1, 120.9, 117.7, 87.6, 85.9, 75.3, 43.1, 26.6. IR: 3399, 2925, 1708, 1603, 1478, 1352, 1307, 1140, 1090, 1015, 829, 755, 698, 526. HRMS-ESI (m/z) $[\mathrm{M}+\mathrm{H}]^{+}$calcd for $\mathrm{C}_{24} \mathrm{H}_{17} \mathrm{CINO}$ : 370.0993; Found, 370.0992.

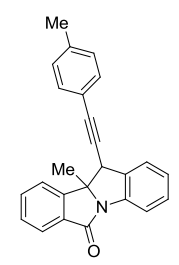

(10bS, 11R)-10b-methyl-11-(p-tolylethynyl)-10b,11-dihydro-6H-isoindolo[2,1-a]indol-6-one (3h): brown solid (96\%, $67.0 \mathrm{mg})$; m.p. $62-64{ }^{\circ} \mathrm{C} ;{ }^{1} \mathrm{H}$ NMR $\left(\mathrm{CDCl}_{3}, 400 \mathrm{MHz}\right) \delta: 7.89(\mathrm{~d}, J=7.6 \mathrm{~Hz}, 1 \mathrm{H}), 7.73(\mathrm{~d}, J=7.6 \mathrm{~Hz}, 1 \mathrm{H})$, 7.56-7.64 (m, 2H), 7.48-7.52 (m, 1H), $7.44(\mathrm{~d}, J=7.6 \mathrm{~Hz}, 1 \mathrm{H}), 7.35-7.29(\mathrm{~m}, 1 \mathrm{H}), 7.14-7.18(\mathrm{~m}, 1 \mathrm{H}), 6.87(\mathrm{~d}, J=$ $8.0 \mathrm{~Hz}, 2 \mathrm{H}), 6.66(\mathrm{~d}, J=8.0 \mathrm{~Hz}, 2 \mathrm{H}), 4.21(\mathrm{~s}, 1 \mathrm{H}), 2.20(\mathrm{~s}, 3 \mathrm{H}), 1.68(\mathrm{~s}, 3 \mathrm{H}) .{ }^{13} \mathrm{C} \mathrm{NMR}\left(100 \mathrm{MHz}, \mathrm{CDCl}_{3}\right) \delta$ : 168.6, 149.1, 138.7, 137.9, 135.8, 132.7, 132.4, 131.0, 129.0, 128.7, 128.6, 126.0, 124.9, 124.5, 123.1, 119.3, 117.6, 87.0, 85.9, 75.2, 43.1, 26.6, 21.3. IR: 3399, 2925, 1707, 1603, 1510, 1478, 1353, 1307, 1140, 817, 756, 680, 529. HRMS-ESI (m/z) $[\mathrm{M}+\mathrm{H}]^{+}$calcd for $\mathrm{C}_{25} \mathrm{H}_{20} \mathrm{NO}$ : 350.1539; Found, 350.1538.

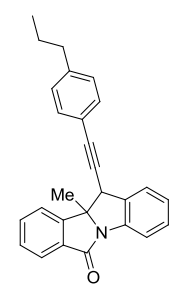

(10bS，11R)-10b-methyl-11-((4-propylphenyl)ethynyl)-10b,11-dihydro-6H-isoindolo[2,1-a]indol-6-one (3i): brown solid (85\%, $64.1 \mathrm{mg})$; m.p. 104-106 ${ }^{\circ} \mathrm{C} ;{ }^{1} \mathrm{H} \mathrm{NMR}\left(\mathrm{CDCl}_{3}, 400 \mathrm{MHz}\right) \delta: 7.90(\mathrm{~d}, J=7.6 \mathrm{~Hz}, 1 \mathrm{H}), 7.73(\mathrm{~d}, J=$ 8.0 Hz, 1H), 7.63-7.66 (m, 1H), 7.53-7.62 (m, 1H), 7.49-7.51 (m, 1H), $7.44(\mathrm{~d}, J=7.6 \mathrm{~Hz}, 1 \mathrm{H}), 7.35-7.39(\mathrm{~m}, 1 \mathrm{H})$, 7.14-7.18 (m, 1H), $6.88(\mathrm{~d}, J=8.4 \mathrm{~Hz}, 2 \mathrm{H}), 6.69(\mathrm{~d}, J=8.4 \mathrm{~Hz}, 2 \mathrm{H}), 4.21(\mathrm{~s}, 1 \mathrm{H}), 2.44(\mathrm{t}, J=7.6 \mathrm{~Hz}, 2 \mathrm{H}), 1.69$ (s, $3 \mathrm{H}), 1.48-1.54(\mathrm{~m}, 2 \mathrm{H}), 0.84(\mathrm{t}, J=7.2 \mathrm{~Hz}, 3 \mathrm{H}) .{ }^{13} \mathrm{C} \mathrm{NMR}\left(100 \mathrm{MHz}, \mathrm{CDCl}_{3}\right) \delta: 168.6,149.1,142.7,138.7,135.8$, 132.8, 132.4, 131.0, 129.0, 128.7, 128.0, 126.0, 124.9, 124.5, 123.1, 119.6, 117.6, 87.1, 85.9, 75.2, 43.2, 37.7, 26.7, 24.2, 13.6. IR: 3402, 2961, 2870, 1708, 1603, 1510, 1478, 1355, 1307, 1214, 1141, 1022, 842, 755, 702, 597, 538. HRMS-ESI (m/z) $[\mathrm{M}+\mathrm{H}]^{+}$calcd for $\mathrm{C}_{27} \mathrm{H}_{24} \mathrm{NO}$ : 378.1852; Found, 378.1858 . 


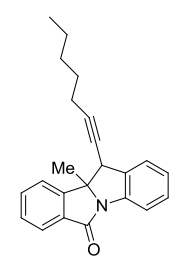

(10bS, 11R)-11-(hept-1-yn-1-yl)-10b-methyl-10b,11-dihydro-6H-isoindolo[2,1-a]indol-6-one (3k): brown oil $(93 \%, 61.2 \mathrm{mg}) ;{ }^{1} \mathrm{H} \mathrm{NMR}\left(\mathrm{CDCl}_{3}, 400 \mathrm{MHz}\right) \delta: 7.86(\mathrm{~d}, J=7.2 \mathrm{~Hz}, 1 \mathrm{H}), 7.70(\mathrm{~d}, J=7.6 \mathrm{~Hz}, 1 \mathrm{H}), 7.62(\mathrm{t}, J=7.2$ $\mathrm{Hz}, 1 \mathrm{H}), 7.46-7.53(\mathrm{~m}, 2 \mathrm{H}), 7.36(\mathrm{t}, J=7.6 \mathrm{~Hz}, 2 \mathrm{H}), 7.14(\mathrm{t}, J=7.2 \mathrm{~Hz}, 1 \mathrm{H}), 3.97(\mathrm{~s}, 1 \mathrm{H}), 1.69-1.73(\mathrm{~m}, 2 \mathrm{H}), 1.61$ $(\mathrm{s}, 3 \mathrm{H}), 1.02-1.08(\mathrm{~m}, 2 \mathrm{H}), 0.88-0.94(\mathrm{~m}, 2 \mathrm{H}), 0.83-0.86(\mathrm{~m}, 2 \mathrm{H}), 0.76(\mathrm{t}, J=7.2 \mathrm{~Hz}, 3 \mathrm{H}) .{ }^{13} \mathrm{C} \mathrm{NMR}(100 \mathrm{MHz}$, $\mathrm{CDCl}_{3}$ ) $\delta: 168.4,149.2,138.4,136.8,132.9,132.2,128.7,128.5,125.8,124.8,124.4,123.1,117.5,87.6,77.3$, 75.1, 42.7, 30.4, 27.8, 26.5, 22.0, 18.3, 13.8. IR: 3402, 3049, 2929, 2858, 1709, 1604, 1479, 1354, 1306, 1218, 1141, 1080, 1021, 754, 695, 597. HRMS-ESI (m/z) [M + H] $]^{+}$calcd for $\mathrm{C}_{22} \mathrm{H}_{24} \mathrm{NO}$ : 330.1852; Found, 330.1859.

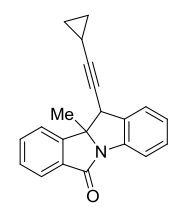

(10bS, 11R)-11-(cyclopropylethynyl)-10b-methyl-10b,11-dihydro-6H-isoindolo[2,1-a]indol-6-one (3l): brown solid $(92 \%, 55.0 \mathrm{mg})$; m.p. $142-144{ }^{\circ} \mathrm{C} ;{ }^{1} \mathrm{H}$ NMR $\left(\mathrm{CDCl}_{3}, 400 \mathrm{MHz}\right) \delta: 7.86(\mathrm{~d}, J=7.6 \mathrm{~Hz}, 1 \mathrm{H}), 7.69(\mathrm{~d}, J=7.6$ $\mathrm{Hz}, 1 \mathrm{H}), 7.62(\mathrm{t}, J=7.2 \mathrm{~Hz}, 1 \mathrm{H}), 7.50$ (t, $J=7.6 \mathrm{~Hz}, 2 \mathrm{H}), 7.35$ (t, $J=7.6 \mathrm{~Hz}, 2 \mathrm{H}), 7.14(\mathrm{t}, J=7.6 \mathrm{~Hz}, 1 \mathrm{H}), 3.93$ (s, 1H), $1.60(\mathrm{~s}, 3 \mathrm{H}), 0.75$ (d, $J=4.4 \mathrm{~Hz}, 1 \mathrm{H}) .0 .30-0.43(\mathrm{~m}, 2 \mathrm{H}),-0.04-0.00(\mathrm{~m}, 1 \mathrm{H}),-0.15(\mathrm{~s}, 1 \mathrm{H}) .{ }^{13} \mathrm{C}$ NMR $(100$ $\left.\mathrm{MHz}, \mathrm{CDCl}_{3}\right) \delta: 168.6,149.2,138.5,136.5,132.8,132.1,128.8,128.5,125.8,124.8,124.3,123.0,117.5,90.7$, 75.3, 72.4, 42.6, 26.3, 7.8, -1.0. IR: 3406, 2928, 1710, 1601, 1473, 1348, 1303, 1138, 883, 754, 691, 596, 518, 469. HRMS-ESI (m/z) $[\mathrm{M}+\mathrm{H}]^{+}$calcd for $\mathrm{C}_{21} \mathrm{H}_{18} \mathrm{NO}$ : 300.1383; Found, 300.1381 .

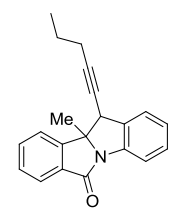

(10bS， 11S)-10b-methyl-11-(pent-1-yn-1-yl)-10b,11-dihydro-6H-isoindolo[2,1-a]indol-6-one (3m): yellow solid (73\%, $43.9 \mathrm{mg})$; m.p. 102-104 ${ }^{\circ} \mathrm{C} ;{ }^{1} \mathrm{H}$ NMR $\left(\mathrm{CDCl}_{3}, 400 \mathrm{MHz}\right) \delta: 7.86(\mathrm{~d}, J=7.6 \mathrm{~Hz}, 1 \mathrm{H}), 7.70(\mathrm{~d}, J=7.6$ $\mathrm{Hz}, 1 \mathrm{H}), 7.62$ (t, $J=7.6 \mathrm{~Hz}, 1 \mathrm{H}), 7.47-7.53(\mathrm{~m}, 2 \mathrm{H}), 7.36(\mathrm{~d}, J=8.0 \mathrm{~Hz}, 2 \mathrm{H}), 7.14(\mathrm{t}, J=7.6 \mathrm{~Hz}, 1 \mathrm{H}), 3.97(\mathrm{~s}, 1 \mathrm{H})$, 1.69-1.72 (m, 2H), $1.62(\mathrm{~s}, 3 \mathrm{H}), 0.91-1.00(\mathrm{~m}, 2 \mathrm{H}), 0.49(\mathrm{t}, J=7.6 \mathrm{~Hz}, 3 \mathrm{H}) .{ }^{13} \mathrm{C} \mathrm{NMR}\left(100 \mathrm{MHz}, \mathrm{CDCl}_{3}\right) \delta: 168.5$, 149.2, 138.4, 136.8, 132.9, 132.3, 128.8, 128.5, 125.8, 124.8, 124.4, 123.1, 117.5, 87.4, 77.5, 75.2, 42.7, 26.6, 21.6, 20.3, 13.0. IR: $3400,2926,1706,1603,1478,1353,1305,1139,754,694,597$. HRMS-ESI (m/z) $[\mathrm{M}+\mathrm{H}]^{+}$calcd for $\mathrm{C}_{21} \mathrm{H}_{20} \mathrm{NO}$ : 302.1539; Found, 302.1537.

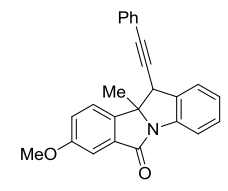

(10bS, 11S)-8-methoxy-10b-methyl-11-(phenylethynyl)-10b,11-dihydro-6H-isoindolo[2,1-a]indol-6-one (3n): 
brown solid (96\%, $70.1 \mathrm{mg})$; m.p. $64-66{ }^{\circ} \mathrm{C} ;{ }^{1} \mathrm{H} \mathrm{NMR}\left(\mathrm{CDCl}_{3}, 400 \mathrm{MHz}\right) \delta: 7.72(\mathrm{~d}, J=6.4 \mathrm{~Hz}, 1 \mathrm{H}), 7.44(\mathrm{~d}, J=$ $7.6 \mathrm{~Hz}, 2 \mathrm{H}), 7.36(\mathrm{~s}, 2 \mathrm{H}), 7.08-7.18(\mathrm{~m}, 5 \mathrm{H}), 6.84(\mathrm{~s}, 2 \mathrm{H}), 4.19(\mathrm{~s}, 1 \mathrm{H}), 3.86(\mathrm{~s}, 3 \mathrm{H}), 1.66(\mathrm{~s}, 3 \mathrm{H}) .{ }^{13} \mathrm{C}$ NMR $(100$ $\left.\mathrm{MHz}, \mathrm{CDCl}_{3}\right) \delta: 168.5,160.4,141.5,138.6,135.8,134.1,131.2,129.0,127.9,126.0,124.9,123.9,122.5,120.8$, 117.5, 107.0, 86.9, 86.7, 74.9, 55.7, 43.1, 26.8. IR: 3404, 2924, 1704, 1602, 1477, 1355, 1280, 1231, 1134, 1057 , 1024, 753, 692. HRMS-ESI (m/z) [M + H] calcd for $\mathrm{C}_{25} \mathrm{H}_{20} \mathrm{NO}_{2}$ : 366.1489; Found, 366.1491.

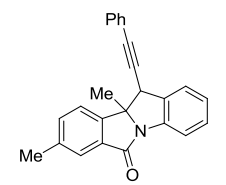

(10bS, 11S)-8,10b-dimethyl-11-(phenylethynyl)-10b,11-dihydro-6H-isoindolo[2,1-a]indol-6-one (3o): brown solid (93\%, 64.9 mg); m.p. 68-70 ${ }^{\circ} \mathrm{C} ;{ }^{1} \mathrm{H} \mathrm{NMR}\left(\mathrm{CDCl}_{3}, 400 \mathrm{MHz}\right) \delta: 7.71$ (t, $\left.J=7.6 \mathrm{~Hz}, 2 \mathrm{H}\right), 7.40$ (d, $J=7.6 \mathrm{~Hz}$, $3 \mathrm{H}), 7.37(\mathrm{t}, J=7.2 \mathrm{~Hz}, 1 \mathrm{H}), 7.08-7.17(\mathrm{~m}, 4 \mathrm{H}), 6.79(\mathrm{~d}, J=7.2 \mathrm{~Hz}, 2 \mathrm{H}), 4.20(\mathrm{~s}, 1 \mathrm{H}), 2.45(\mathrm{~s}, 3 \mathrm{H}), 1.67$ (s, 3H). ${ }^{13} \mathrm{C}$ NMR $\left(100 \mathrm{MHz}, \mathrm{CDCl}_{3}\right) \delta: 168.7,146.4,138.8,138.8,135.7,133.5,132.9,131.2,129.0,127.9,126.0,124.9$, 124.7, 122.8, 122.5, 117.6, 86.8, 86.8, 75.0, 43.1, 26.8, 21.3. IR: 3399, 3049, 2924, 1707, 1601, 1477, 1353, 1307, 1133, 753, 692, 527. HRMS-ESI (m/z) $[\mathrm{M}+\mathrm{H}]^{+}$calcd for $\mathrm{C}_{25} \mathrm{H}_{20} \mathrm{NO}$ : 350.1539; Found, 350.1540.

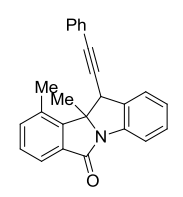

(10bS, 11S)-10,10b-dimethyl-11-(phenylethynyl)-10b,11-dihydro-6H-isoindolo[2,1-a]indol-6-one (3p): brown solid (80\%, $55.8 \mathrm{mg})$; m.p. 138-140 ${ }^{\circ} \mathrm{C} ;{ }^{1} \mathrm{H}$ NMR $\left(\mathrm{CDCl}_{3}, 400 \mathrm{MHz}\right) \delta: 7.74(\mathrm{~d}, J=7.2 \mathrm{~Hz}, 2 \mathrm{H}), 7.46(\mathrm{~d}, J=7.6$ $\mathrm{Hz}, 1 \mathrm{H}), 7.36-7.42(\mathrm{~m}, 3 \mathrm{H}), 7.11-7.18(\mathrm{~m}, 2 \mathrm{H}), 7.07$ (t, $J=7.2 \mathrm{~Hz}, 2 \mathrm{H}), 6.78(\mathrm{~d}, J=7.2 \mathrm{~Hz}, 2 \mathrm{H}), 4.29$ (s, $1 \mathrm{H}), 2.58$ (s, 3H), 1.72 (s, 3H). ${ }^{13} \mathrm{C}$ NMR (100 MHz, $\left.\mathrm{CDCl}_{3}\right) \delta: 167.9,147.0,138.1,135.4,134.2,133.4,133.2,131.2,129.1$, 129.0, 127.9, 126.0, 124.8, 122.5, 122.3, 117.3, 86.2, 86.1, 75.5, 42.3, 24.8, 18.7. IR: 3398, 2926, 1707, 1602, 1479, 1360, 1309, 1139, 1078, 755, 691. HRMS-ESI $(\mathrm{m} / \mathrm{z})[\mathrm{M}+\mathrm{H}]^{+}$calcd for $\mathrm{C}_{25} \mathrm{H}_{20} \mathrm{NO}$ : 350.1539; Found, 350.1544 .

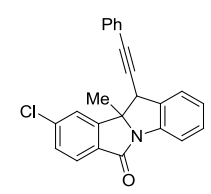

(10bS, 11S)-9-chloro-10b-methyl-11-(phenylethynyl)-10b,11-dihydro-6H-isoindolo[2,1-a]indol-6-one (3q): yellow solid (62\%, $45.8 \mathrm{mg})$; m.p. 170-172 ${ }^{\circ} \mathrm{C} ;{ }^{1} \mathrm{H}$ NMR $\left(\mathrm{CDCl}_{3}, 400 \mathrm{MHz}\right) \delta: 7.81(\mathrm{~d}, J=8.0 \mathrm{~Hz}, 1 \mathrm{H}), 7.72(\mathrm{~d}, J$ $=7.6 \mathrm{~Hz}, 1 \mathrm{H}), 7.60(\mathrm{~d}, J=1.6 \mathrm{~Hz}, 1 \mathrm{H}), 7.49-7.51(\mathrm{~m}, 1 \mathrm{H}), 7.56(\mathrm{~d}, J=7.6 \mathrm{~Hz}, 1 \mathrm{H}), 7.39(\mathrm{~d}, J=7.6 \mathrm{~Hz}, 1 \mathrm{H})$, 7.17-7.21 (m, 2H), 7.10-7.15 (m, 2H), $6.86(\mathrm{t}, J=7.2 \mathrm{~Hz} 2 \mathrm{H}), 4.22(\mathrm{~s}, 1 \mathrm{H}), 1.70(\mathrm{~s}, 3 \mathrm{H}) .{ }^{13} \mathrm{C}$ NMR $(100 \mathrm{MHz}$, $\left.\mathrm{CDCl}_{3}\right) \delta: 167.5,150.6,138.9,138.5,135.3,131.3,131.2,129.4,129.2,128.1,128.0,126.1,125.8,125.2,123.7$, 122.2, 117.6, 87.4, 86.2, 74.9, 43.1, 26.7. IR: 3400, 2925, 1709, 1604, 1479, 1352, 1322, 1140, 1081, 756, 690. HRMS-ESI (m/z) $[\mathrm{M}+\mathrm{H}]^{+}$calcd for $\mathrm{C}_{24} \mathrm{H}_{17} \mathrm{ClNO}$ : 370.0993; Found, 370.0992. 


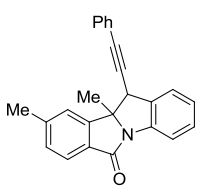

(10bS, 11S)-9,10b-dimethyl-11-(phenylethynyl)-10b,11-dihydro-6H-isoindolo[2,1-a]indol-6-one (3r): yellow solid (71\%, $49.6 \mathrm{mg})$; m.p. $164-166{ }^{\circ} \mathrm{C} ;{ }^{1} \mathrm{H} \mathrm{NMR}\left(\mathrm{CDCl}_{3}, 400 \mathrm{MHz}\right) \delta: 7.77(\mathrm{~d}, J=8.0 \mathrm{~Hz}, 1 \mathrm{H}), 7.71(\mathrm{~d}, J=8.0 \mathrm{~Hz}$, $1 \mathrm{H}), 7.44(\mathrm{~d}, J=7.2 \mathrm{~Hz}, 1 \mathrm{H}), 7.38(\mathrm{~d}, J=7.6 \mathrm{~Hz}, 2 \mathrm{H}), 7.33(\mathrm{t}, J=8.0 \mathrm{~Hz}, 1 \mathrm{H}), 7.12-7.18(\mathrm{~m}, 2 \mathrm{H}), 7.08(\mathrm{t}, J=7.6$ $\mathrm{Hz}, 2 \mathrm{H}), 6.79$ (d, J = 7.2 Hz, 2H), 4.20 (s, 1H), 2.48 (s, 3H), 1.68 (s, 3H). ${ }^{13} \mathrm{C} \mathrm{NMR}\left(100 \mathrm{MHz}, \mathrm{CDCl}_{3}\right) \delta: 168.8$, 149.5, 143.3, 138.9, 135.5, 131.1, 130.2, 129.7, 129.0, 127.9, 126.0, 124.8, 124.4, 123.6, 122.5, 117.6, 86.9, 86.8, 75.0, 43.1, 26.8, 22.0. IR: 3375, 3051, 2925, 1700, 1601, 1478, 1352, 1140, 1079, 837, 752, 696, 605, 537, 482. HRMS-ESI (m/z) $[\mathrm{M}+\mathrm{H}]^{+}$calcd for $\mathrm{C}_{25} \mathrm{H}_{20} \mathrm{NO}$ : 350.1539; Found, 350.1538.

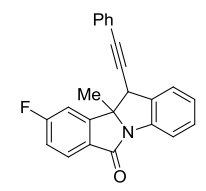

(10bS, 11S)-9-fluoro-10b-methyl-11-(phenylethynyl)-10b,11-dihydro-6H-isoindolo[2,1-a]indol-6-one (3s): yellow solid (51\%, $36.0 \mathrm{mg})$; m.p. $168-170{ }^{\circ} \mathrm{C} ;{ }^{1} \mathrm{H}$ NMR $\left(\mathrm{CDCl}_{3}, 400 \mathrm{MHz}\right) \delta$ : 7.86-7.89 (m, 1H), 7.71 (d, $J=7.6$ $\mathrm{Hz}, 1 \mathrm{H}), 7.45$ (d, $J=7.2 \mathrm{~Hz}, 1 \mathrm{H}), 7.38$ (t, $J=7.6 \mathrm{~Hz}, 1 \mathrm{H}), 7.23-7.29(\mathrm{~m}, 1 \mathrm{H}), 7.08-7.19(\mathrm{~m}, 5 \mathrm{H}), 6.85$ (d, $J=7.2$ $\mathrm{Hz}, 2 \mathrm{H}), 4.22$ (s, 1H), 1.69 (s, 3H). ${ }^{13} \mathrm{C}$ NMR (100 MHz, $\left.\mathrm{CDCl}_{3}\right) \delta: 167.5,167.0,164.4,151.7,151.6,138.6,135.3$, 131.1, 129.2, 128.8, 128.1, 128.0, 126.7, 126.7, 126.0, 125.1, 122.3, 117.6, 116.7, 116.4, 110.7, 110.5, 87.2, 86.1, 74.8, 43.1, 26.6. IR: 3401, 3062, 2925, 1712, 1600, 1478, 1352, 1308, 1191, 1140, 938, 753, 692. HRMS-ESI (m/z) $[\mathrm{M}+\mathrm{H}]^{+}$calcd for $\mathrm{C}_{24} \mathrm{H}_{17} \mathrm{FNO}$ : 354.1289; Found, 354.1291 .

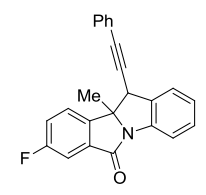

(10bS， 11S)-8-fluoro-10b-methyl-11-(phenylethynyl)-10b,11-dihydro-6H-isoindolo[2,1-a]indol-6-one (3t): yellow solid $(61 \%$, $43.1 \mathrm{mg})$; m.p. $158-160{ }^{\circ} \mathrm{C} ;{ }^{1} \mathrm{H}$ NMR $\left(\mathrm{CDCl}_{3}, 400 \mathrm{MHz}\right) \delta: 7.72(\mathrm{~d}, J=8.0 \mathrm{~Hz}, 1 \mathrm{H}), 7.54-7.57$ (m, 2H), $7.45(\mathrm{~d}, J=7.2 \mathrm{~Hz}, 1 \mathrm{H}), 7.31-7.41(\mathrm{~m}, 2 \mathrm{H}), 7.09-7.21(\mathrm{~m}, 4 \mathrm{H}), 6.83(\mathrm{~d}, J=6.8 \mathrm{~Hz}, 2 \mathrm{H}), 4.22(\mathrm{~s}, 1 \mathrm{H})$, 1.69 (s, 3H). ${ }^{13} \mathrm{C}$ NMR (100 MHz, $\left.\mathrm{CDCl}_{3}\right) \delta: 167.2,164.4,161.9,144.6,138.4,135.7,135.1,135.0,131.1,129.2$, 128.1, 128.0, 126.1, 125.2, 124.7, 124.7, 122.3, 120.0, 119.8, 117.7, 111.3, 111.0, 87.2, 86.3, 75.0, 43.2, 26.7. IR: 3407, 3059, 2925, 1710, 1604, 1477, 1356, 1267, 1220, 1125, 753, 691, 589, 542. HRMS-ESI (m/z) $[\mathrm{M}+\mathrm{H}]^{+}$ calcd for $\mathrm{C}_{24} \mathrm{H}_{17} \mathrm{FNO}$ 354.1289; Found, 354.1290.

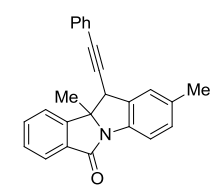

(10bS, 11S)-2,10b-dimethyl-11-(phenylethynyl)-10b,11-dihydro-6H-isoindolo[2,1-a]indol-6-one (3u): yellow solid (53\%, 37.0 mg); m.p. 148-150 ${ }^{\circ} \mathrm{C} ;{ }^{1} \mathrm{H}$ NMR $\left(\mathrm{CDCl}_{3}, 400 \mathrm{MHz}\right) \delta: 7.89(\mathrm{~d}, J=7.6 \mathrm{~Hz}, 1 \mathrm{H}), 7.56-7.65(\mathrm{~m}, 3 \mathrm{H})$, 
$7.51(\mathrm{t}, J=7.6 \mathrm{~Hz}, 1 \mathrm{H}), 7.25(\mathrm{~s}, 1 \mathrm{H}), 7.18(\mathrm{~d}, J=8.0 \mathrm{~Hz}, 1 \mathrm{H}), 7.05-7.15(\mathrm{~m}, 3 \mathrm{H}), 6.77(\mathrm{~d}, J=7.2 \mathrm{~Hz}, 2 \mathrm{H}), 4.17$ (s, 1H), 2.37 (s, 3H), 1.69 (s, 3H). ${ }^{13} \mathrm{C}$ NMR (100 MHz, $\left.\mathrm{CDCl}_{3}\right) \delta: 168.5,149.0,136.3,135.7,134.7,132.9,132.3$, 131.2, 129.6, 128.7, 127.9, 126.6, 124.5, 123.1, 122.5, 117.3, 86.8, 86.7, 75.4, 43.1, 26.6, 21.2. IR: 3393, 3054, 2924, 1705, 1610, 1490, 1352, 1303, 1094, 817, 759, 693. HRMS-ESI (m/z) $[\mathrm{M}+\mathrm{H}]^{+}$calcd for $\mathrm{C}_{25} \mathrm{H}_{20} \mathrm{NO}$ : 350.1539; Found, 350.1545.

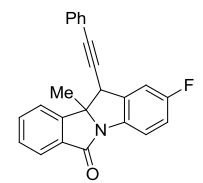

(10bS, 11S)-2-fluoro-10b-methyl-11-(phenylethynyl)-10b,11-dihydro-6H-isoindolo[2,1-a]indol-6-one (3v): yellow solid (28\%, $19.8 \mathrm{mg})$; m.p. $164-166{ }^{\circ} \mathrm{C} ;{ }^{1} \mathrm{H} \mathrm{NMR}\left(\mathrm{CDCl}_{3}, 400 \mathrm{MHz}\right) \delta: 7.90(\mathrm{~d}, J=7.6 \mathrm{~Hz}, 1 \mathrm{H}), 7.63-7.68$ (m, 2H), 7.51-7.59 (m, 2H), 7.13-7.19(m, 2H), 7.06-7.10 (m, 3H), 6.77 (d, J = 6.8 Hz, 2H), 4.20 (s, 1H), 1.70 (s, $3 \mathrm{H}) .{ }^{13} \mathrm{C}$ NMR $\left(100 \mathrm{MHz}, \mathrm{CDCl}_{3}\right) \delta: 168.7,161.5,159.0,148.8,137.4,137.4,134.9,132.6,132.5,131.1,128.9$, 128.1, 127.9, 124.6, 123.1, 122.1, 118.5, 118.4, 115.8, 115.6, 113.6, 113.4, 87.5, 85.8, 75.7, 43.3, 26.5. IR: 3398, 3056, 2925, 1709, 1613, 1484, 1357, 1305, 1263, 1226, 1133, 819, 758, 693, 529. HRMS-ESI (m/z) $[\mathrm{M}+\mathrm{H}]^{+}$ calcd for $\mathrm{C}_{24} \mathrm{H}_{17} \mathrm{FNO}$ : 354.1289; Found, 354.1290.

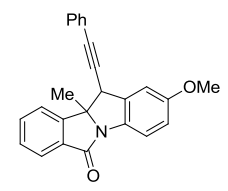

(10bS, 11S)-2-methoxy-10b-methyl-11-(phenylethynyl)-10b,11-dihydro-6H-isoindolo[2,1-a]indol-6-one (3w): brown solid (62\%, $45.3 \mathrm{mg})$; m.p. $122-124{ }^{\circ} \mathrm{C} ;{ }^{1} \mathrm{H} \mathrm{NMR}\left(\mathrm{CDCl}_{3}, 400 \mathrm{MHz}\right) \delta: 7.89(\mathrm{~d}, J=7.6 \mathrm{~Hz}, 1 \mathrm{H}), 7.63(\mathrm{t}, J=$ $8, .4 \mathrm{~Hz}, 2 \mathrm{H}), 7.57(\mathrm{~d}, J=7.6 \mathrm{~Hz}, 1 \mathrm{H}), 7.51(\mathrm{t}, J=7.6 \mathrm{~Hz}, 1 \mathrm{H}), 7.13(\mathrm{t}, J=7.2 \mathrm{~Hz}, 1 \mathrm{H}), 7.07(\mathrm{t}, J=7.2 \mathrm{~Hz}, 2 \mathrm{H})$, $7.02(\mathrm{~d}, J=1.6 \mathrm{~Hz}, 1 \mathrm{H}), 6.91(\mathrm{t}, J=6.8 \mathrm{~Hz}, 1 \mathrm{H}), 6.77(\mathrm{~d}, J=7.2 \mathrm{~Hz}, 2 \mathrm{H}), 4.17(\mathrm{~s}, 1 \mathrm{H}), 3.82(\mathrm{~s}, 3 \mathrm{H}), 1.69$ (s, 3H). ${ }^{13} \mathrm{C}$ NMR $\left(100 \mathrm{MHz}, \mathrm{CDCl}_{3}\right) \delta: 168.6,157.4,148.9,137.1,132.9,132.3,131.2,128.7,127.9,127.9,124.5,123.0$, 122.4, 118.2, 114.0, 112.3, 87.0, 86.4, 75.7, 55.7, 43.4, 26.5. IR: 3393, 2925, 1704, 1596, 1490, 1358, 1308, 1278, 1235, 1137, 1091, 1031, 815, 758, 693. HRMS-ESI $(\mathrm{m} / \mathrm{z})[\mathrm{M}+\mathrm{H}]^{+}$calcd for $\mathrm{C}_{25} \mathrm{H}_{20} \mathrm{NO}_{2}$ : 366.1489; Found, 366.1491

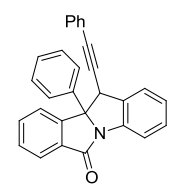

(10bR, 11S)-10b-phenyl-11-(phenylethynyl)-10b,11-dihydro-6H-isoindolo[2,1-a]indol-6-one (3x): yellow solid (76\%, $63.7 \mathrm{mg})$; m.p. 60-62 ${ }^{\circ} \mathrm{C} ;{ }^{1} \mathrm{H}$ NMR $\left(\mathrm{CDCl}_{3}, 400 \mathrm{MHz}\right) \delta: 7.84-7.89(\mathrm{~m}, 2 \mathrm{H}), 7.68-7.73(\mathrm{~m}, 3 \mathrm{H}), 7.56(\mathrm{~d}, J=$ $7.2 \mathrm{~Hz}, 1 \mathrm{H}), 7.45(\mathrm{t}, J=7.6 \mathrm{~Hz}, 1 \mathrm{H}), 7.34-7.38(\mathrm{~m}, 4 \mathrm{H}), 7.30(\mathrm{~d}, J=7.6 \mathrm{~Hz}, 1 \mathrm{H}), 7.07-7.23(\mathrm{~m}, 4 \mathrm{H}), 6.82(\mathrm{~d}, J=$ $6.8 \mathrm{~Hz}, 2 \mathrm{H}), 4.85$ (s, 1H). ${ }^{13} \mathrm{C} \mathrm{NMR}\left(100 \mathrm{MHz}, \mathrm{CDCl}_{3}\right) \delta: 169.1,148.5,142.2,139.2,135.5,132.6,132.2,131.2$, 129.1, 128.9, 128.7, 128.2, 128.0, 127.9, 125.7, 125.3, 125.0, 124.6, 124.1, 122.3, 117.5, 87.8, 86.6, 80.4, 45.2. IR: 3406, 3057, 2924, 1708, 1600, 1478, 1355, 1306, 1141, 1071, 754, 701, 603, 527. HRMS-ESI (m/z) $[\mathrm{M}+\mathrm{Na}]^{+}$ calcd for $\mathrm{C}_{29} \mathrm{H}_{19} \mathrm{NONa}$ : 420.1359; Found, 420.1364 . 


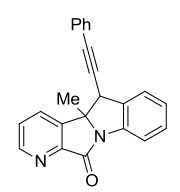

(4bS，5S)-4b-methyl-5-(phenylethynyl)-4bH-pyrido[3',2':3,4]pyrrolo[1,2-a]indol-11(5H)-one (3y): brown solid (41\%, $27.6 \mathrm{mg})$; m.p. 190-192 ${ }^{\circ} \mathrm{C} ;{ }^{1} \mathrm{H}$ NMR $\left(\mathrm{CDCl}_{3}, 400 \mathrm{MHz}\right) \delta: 8.85(\mathrm{~d}, J=3.6 \mathrm{~Hz}, 1 \mathrm{H}), 7.98(\mathrm{~d}, J=7.6$ $\mathrm{Hz}, 1 \mathrm{H}), 7.82(\mathrm{~d}, J=7.6 \mathrm{~Hz}, 1 \mathrm{H}), 7.53(\mathrm{~d}, J=4.8 \mathrm{~Hz}, 1 \mathrm{H}), 7.48(\mathrm{t}, J=8.0 \mathrm{~Hz}, 1 \mathrm{H}), 7.41(\mathrm{t}, J=7.6 \mathrm{~Hz}, 1 \mathrm{H}), 7.21(\mathrm{t}$, $J=7.2 \mathrm{~Hz}, 1 \mathrm{H}), 7.15(\mathrm{~d}, J=6.8 \mathrm{~Hz}, 1 \mathrm{H}), 7.09(\mathrm{t}, J=7.2 \mathrm{~Hz}, 2 \mathrm{H}), 6.81(\mathrm{~d}, J=6.8 \mathrm{~Hz}, 2 \mathrm{H}), 4.29(\mathrm{~s}, 1 \mathrm{H}), 1.72(\mathrm{~s}$, $3 \mathrm{H}) .{ }^{13} \mathrm{C} \mathrm{NMR}\left(100 \mathrm{MHz}, \mathrm{CDCl}_{3}\right) \delta: 166.2,151.6,150.5,143.4,138.2,135.0,131.4,131.1,129.3,128.2,128.0$, 126.0, 125.8, 125.5, 122.0, 118.0, 87.6, 86.0, 73.0, 43.0, 26.4. IR: 3410, 2967, 1712, 1599, 1478, 1353, 1306, 1173, 1119, 1072, 812, 764, 684, 598, 529, 461. HRMS-ESI (m/z) $[\mathrm{M}+\mathrm{H}]^{+}$calcd for $\mathrm{C}_{23} \mathrm{H}_{17} \mathrm{~N}_{2} \mathrm{O}$ : 337.1335; Found, 337.1338 .

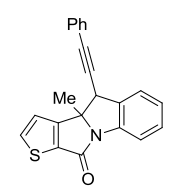

(3bS, 4S)-3b-methyl-4-(phenylethynyl)-3bH-thieno[3',2':3,4]pyrrolo[1,2-a]indol-10(4H)-one (3z): brown solid (50\%, $34.1 \mathrm{mg})$; m.p. $146-148{ }^{\circ} \mathrm{C} ;{ }^{1} \mathrm{H} \mathrm{NMR}\left(\mathrm{CDCl}_{3}, 400 \mathrm{MHz}\right) \delta: 7.75(\mathrm{~d}, J=4.8 \mathrm{~Hz}, 1 \mathrm{H}), 7.65(\mathrm{~d}, J=7.6 \mathrm{~Hz}, 1 \mathrm{H})$, $7.42(\mathrm{~d}, J=7.2 \mathrm{~Hz}, 1 \mathrm{H}), 7.36(\mathrm{t}, J=7.6 \mathrm{~Hz}, 1 \mathrm{H}), 7.11-7.19(\mathrm{~m}, 5 \mathrm{H}), 6.92(\mathrm{~d}, J=7.2 \mathrm{~Hz}, 2 \mathrm{H}), 4.18(\mathrm{~s}, 1 \mathrm{H}), 1.70(\mathrm{~s}$, $3 \mathrm{H}) .{ }^{13} \mathrm{C} \mathrm{NMR}\left(100 \mathrm{MHz}, \mathrm{CDCl}_{3}\right) \delta: 164.8,160.1,139.2,136.8,135.2,135.2,131.3,129.1,128.0,128.0,126.0$, 124.8, 122.5, 121.1, 117.3, 86.6, 86.6, 74.2, 42.8, 26.3. IR: 3380, 3078, 2924, 1703, 1600, 1478, 1341, 1303, 1119, 1070, 910, 801, 758, 737, 690, 641. HRMS-ESI (m/z) [M + H] $]^{+}$calcd for $\mathrm{C}_{22} \mathrm{H}_{16} \mathrm{NOS}: 342.0947$; Found, 342.0948.

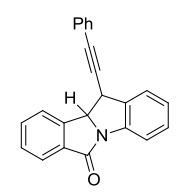

(10bS, 11S)-11-(phenylethynyl)-10b,11-dihydro-6H-isoindolo[2,1-a]indol-6-one (3aa): yellow solid (16\%, 10.3 mg); m.p. 116-118 ${ }^{\circ} \mathrm{C} ;{ }^{1} \mathrm{H} \mathrm{NMR}\left(\mathrm{CDCl}_{3}, 400 \mathrm{MHz}\right) \delta: 7.92(\mathrm{~d}, J=7.6 \mathrm{~Hz}, 1 \mathrm{H}), 7.74(\mathrm{~d}, J=7.6 \mathrm{~Hz}, 1 \mathrm{H}), 7.61-7.67$ $(\mathrm{m}, 2 \mathrm{H}), 7.52-7.56(\mathrm{~m}, 1 \mathrm{H}), 7.45(\mathrm{~d}, J=7.6 \mathrm{~Hz}, 1 \mathrm{H}), 7.37(\mathrm{t}, J=7.6 \mathrm{~Hz}, 1 \mathrm{H}), 7.13-7.18(\mathrm{~m}, 2 \mathrm{H}), 7.09(\mathrm{t}, J=7.2$ $\mathrm{Hz}, 2 \mathrm{H}), 6.83(\mathrm{t}, J=7.2 \mathrm{~Hz}, 2 \mathrm{H}), 5.65(\mathrm{~d}, J=7.6 \mathrm{~Hz}, 1 \mathrm{H}), 4.63(\mathrm{~d}, J=7.6 \mathrm{~Hz}, 1 \mathrm{H}) .{ }^{13} \mathrm{C} \mathrm{NMR}\left(100 \mathrm{MHz}, \mathrm{CDCl}_{3}\right) \delta$ : $168.8,143.6,139.9,135.9,134.4,132.3,131.3,129.1,128.9,128.0,128.0,125.8,124.9,124.6,124.1,122.4$, 116.9, 86.5, 85.9, 68.3, 36.5. IR: 3397, 3056, 2926, 1707, 1601, 1478, 1363, 1208, 1102, 755, 691,528, 492. HRMS-ESI (m/z) $[\mathrm{M}+\mathrm{H}]^{+}$calcd for $\mathrm{C}_{23} \mathrm{H}_{16} \mathrm{NO}$ : 322.1226; Found, 322.1227.

\section{References.}

(1) (a) Petrone, D. A.; Yen, A.; Zeidan, N.; Lautens, M. Org. Lett. 2015, 17, 4838. (b) Shen, C.; Liu, R.-R.; Fan, R.-J.; Li, Y.-L.; Xu, T.-F.; Gao, J.-R.; Jia, Y.-X. J. Am. Chem. Soc. 2015, 137, 4936.

(2) (a) Park, K.; Palani, T.; Pyo, A.; Lee, S. Tetrahedron Lett. 2012, 53, 733. (b) Park, K.; You, J. M.; Jeon, S.; Lee, S. Eur. J. Org. Chem. 2013, 2013, 1973. 


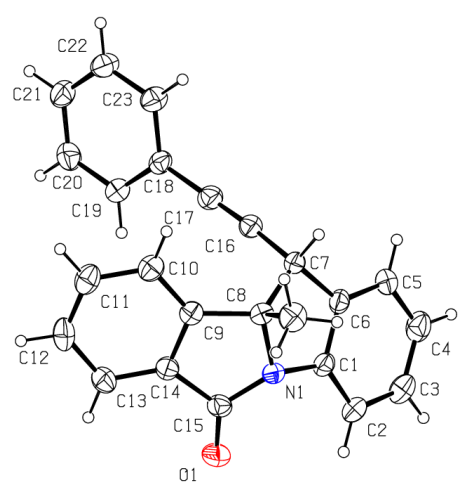

Bond precision: $\quad \mathrm{C}-\mathrm{C}=0.0049 \mathrm{~A}$

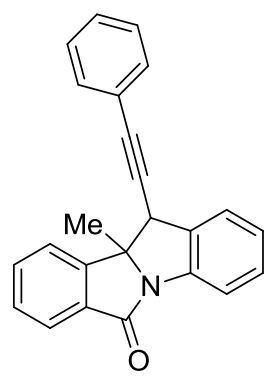

3a

Thermal ellipsoids are shown at $30 \%$ probability.

\begin{tabular}{|c|c|c|}
\hline Cell: & $\begin{array}{l}a=15.3059(8) \\
\text { alpha }=90\end{array}$ & $\begin{array}{l}b=12.1881(6) \\
\text { beta }=90\end{array}$ \\
\hline
\end{tabular}

Temperature: $\quad 294 \mathrm{~K}$

Wavelength $=0.71073$

Calculated Reported

Volume

1796.10(17)

1796.10(17)

Space group

$\mathrm{Pn}$ a 21

$\mathrm{Pn}$ a 21

Hall group

P 2c -2n

$\mathrm{P} 2 \mathrm{c}-2 \mathrm{n}$

Moiety formula

$\mathrm{C} 24 \mathrm{H} 17 \mathrm{~N} \mathrm{O}$

$\mathrm{C} 24 \mathrm{H} 17 \mathrm{~N} \mathrm{O}$

Sum formula

$\mathrm{C} 24 \mathrm{H} 17 \mathrm{~N} \mathrm{O}$

C24 H17 N O

$\mathrm{Mr}$

335.39

335.39

Dx, g cm-3

1.240

1.240

$\mathrm{Z}$

4

4

0.075

0.075

704.0

704.0

704.28

F000'

$18,15,11$

$18,15,11$

3536[ 1879]

2477

Nref

$0.986,0.990$

$0.625,1.000$

Tmin,Tmax

0.984

Correction method $=\#$ Reported T Limits: Tmin $=0.625$ Tmax $=1.000$ AbsCorr $=$ MULTI-SCAN

Data completeness $=1.32 / 0.70$

$\operatorname{Theta}(\max )=26.020$

$\mathrm{R}($ reflections $)=0.0499(1870)$ $\mathrm{wR} 2($ reflections $)=0.1220(2477)$

$\mathrm{S}=1.067$ Npar $=236$ 
8. Copies of ${ }^{1} \mathrm{H}$ and ${ }^{13} \mathrm{C}$ Spectra

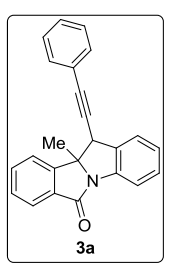

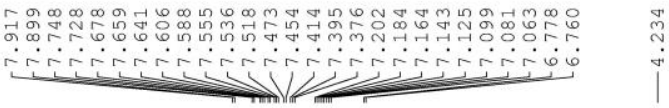
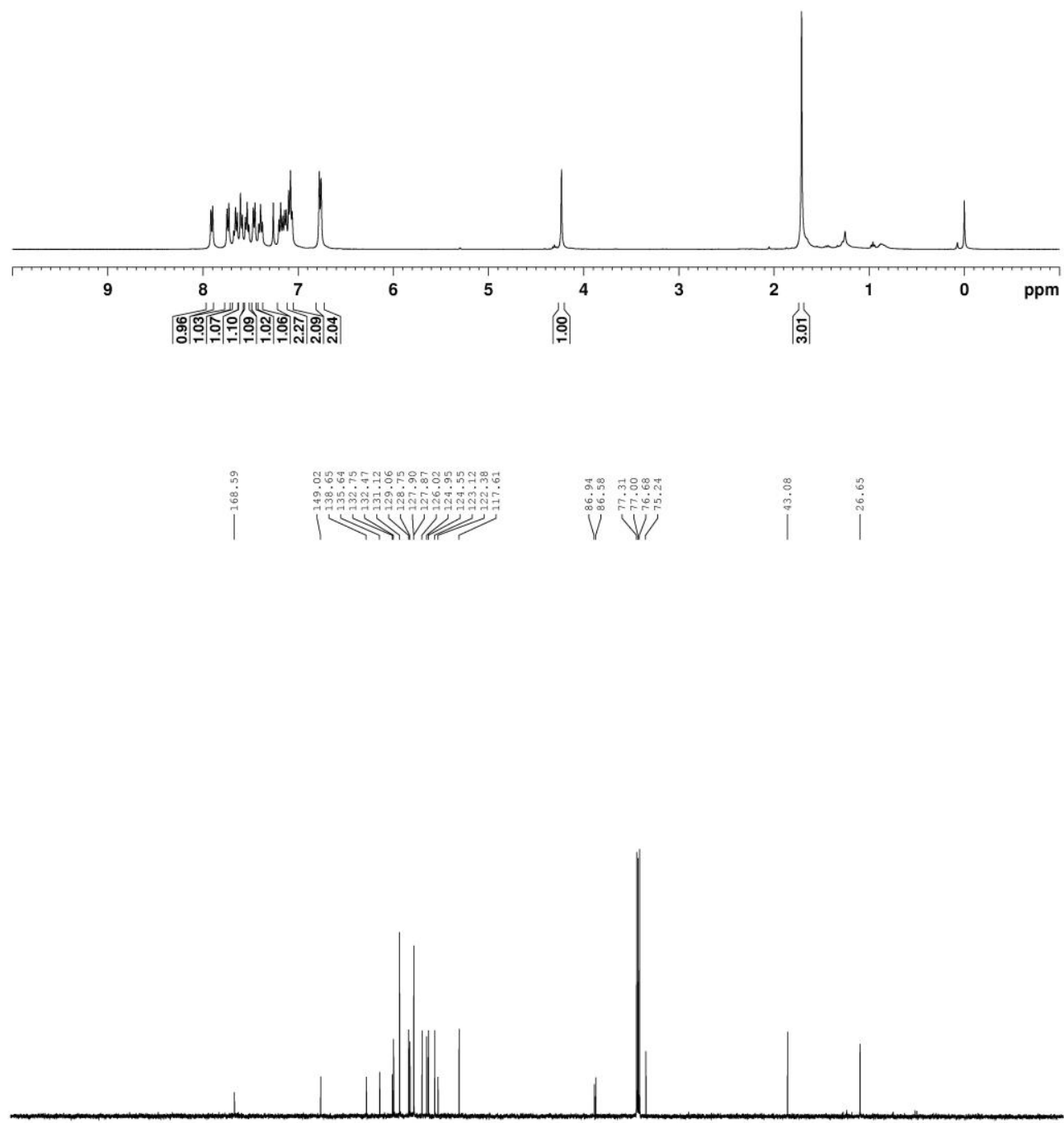

$\begin{array}{lllllllllllllllllllllll}210 & 200 & 190 & 180 & 170 & 160 & 150 & 140 & 130 & 120 & 110 & 100 & 90 & 80 & 70 & 60 & 50 & 40 & 30 & 20 & 10 & 0 & \mathrm{ppm}\end{array}$ 


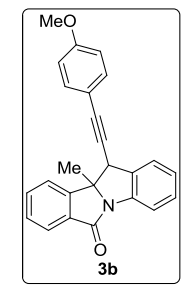

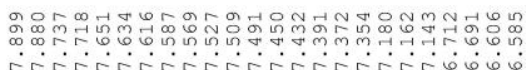

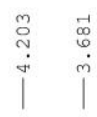
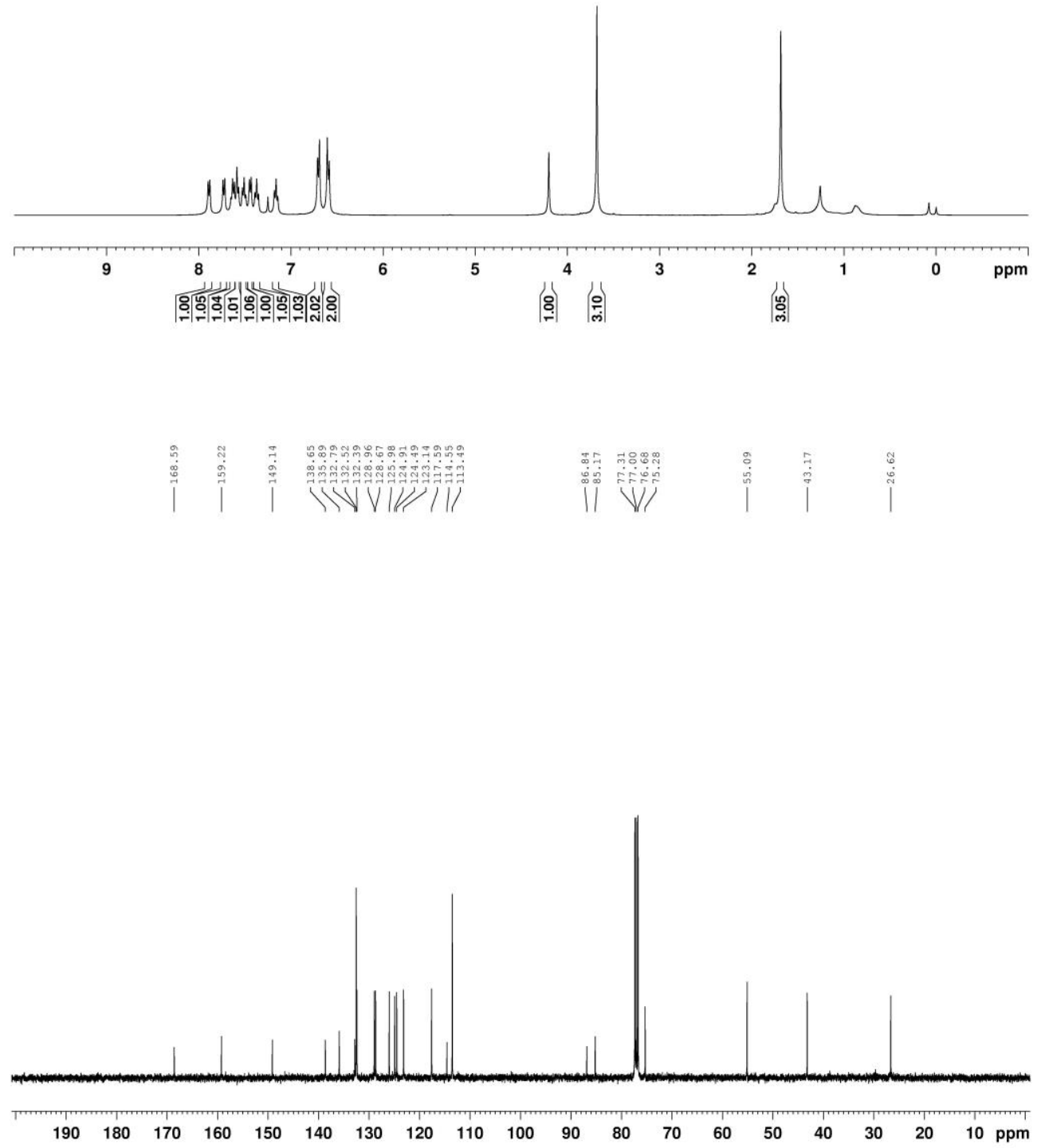


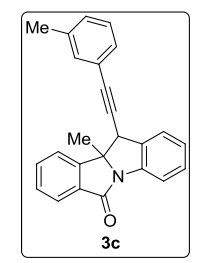

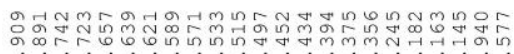

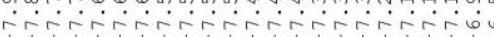

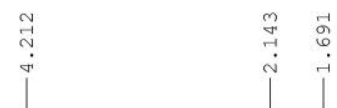

웅

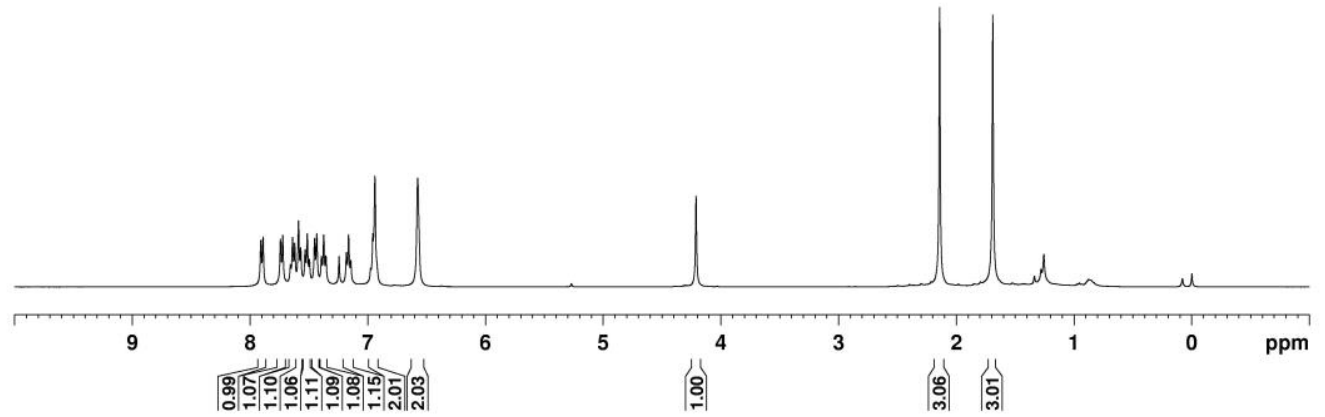

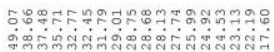

证

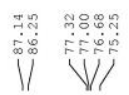

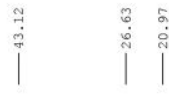

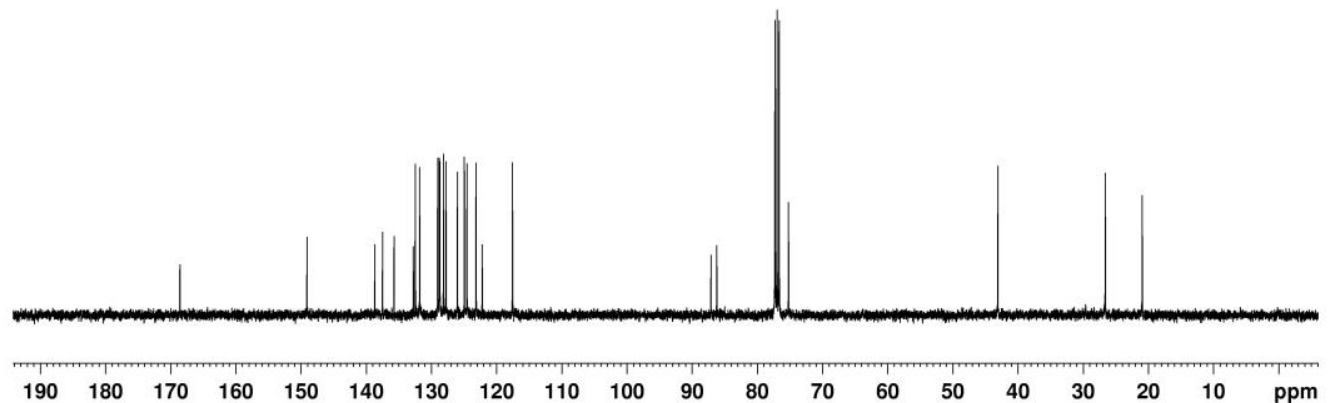




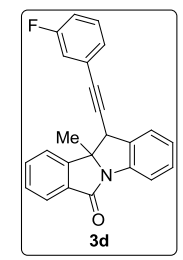

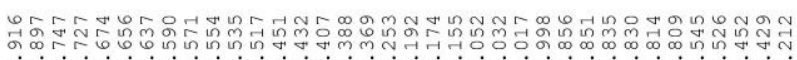

rritro

$\begin{array}{ll}\stackrel{\circ}{\circ} & \stackrel{0}{\circ} \\ \stackrel{0}{-1} & \stackrel{0}{\mid}\end{array}$

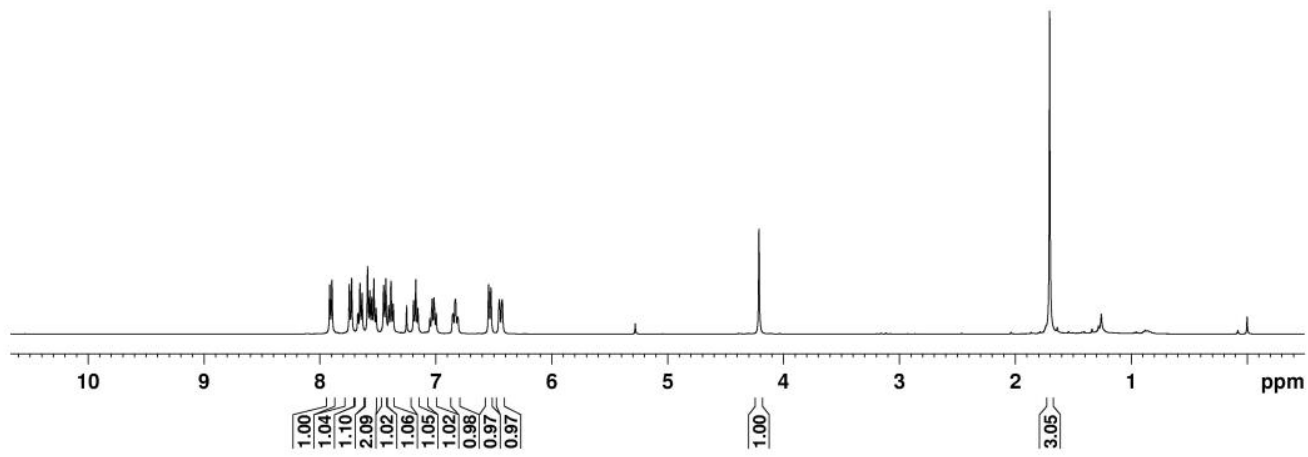

||

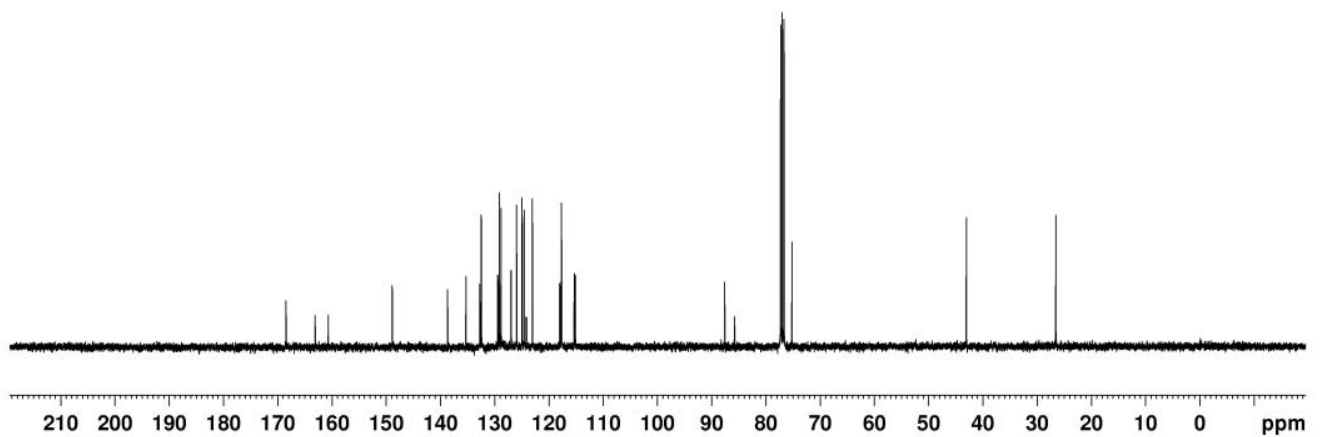




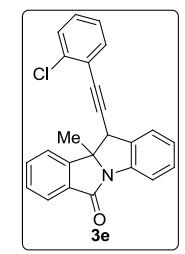

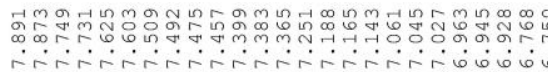

ֻั
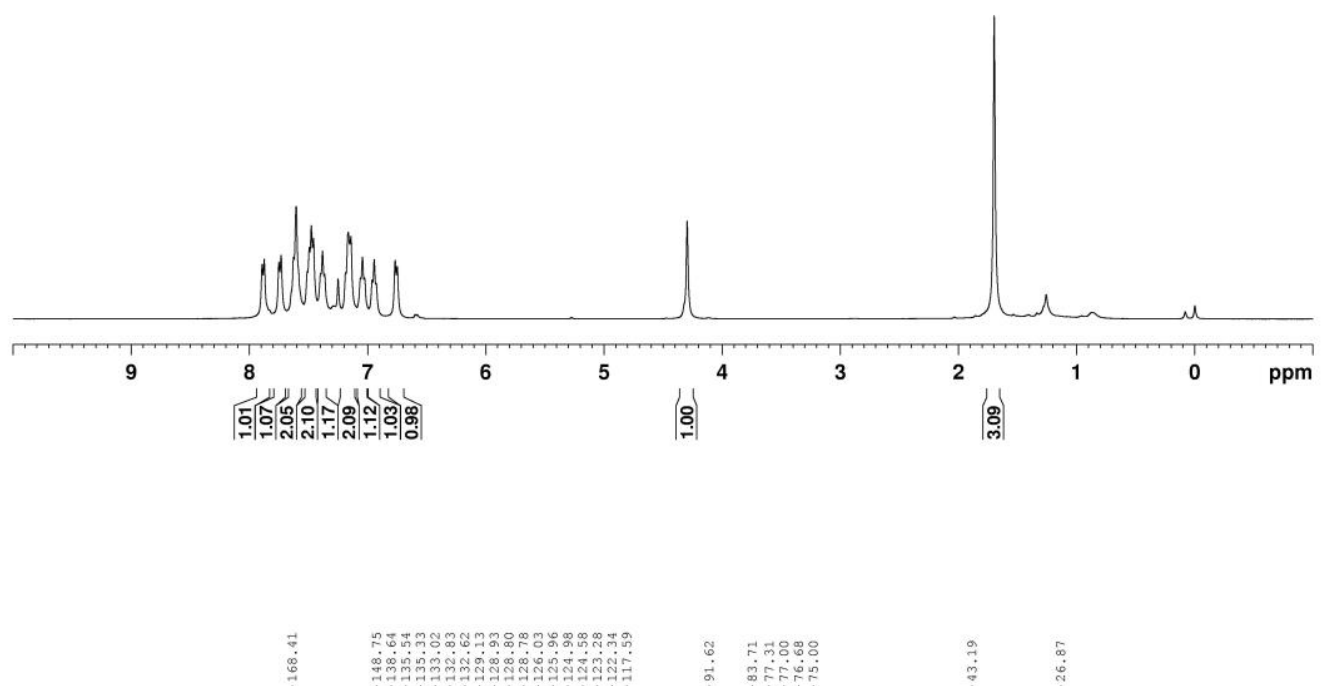

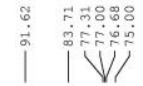

|

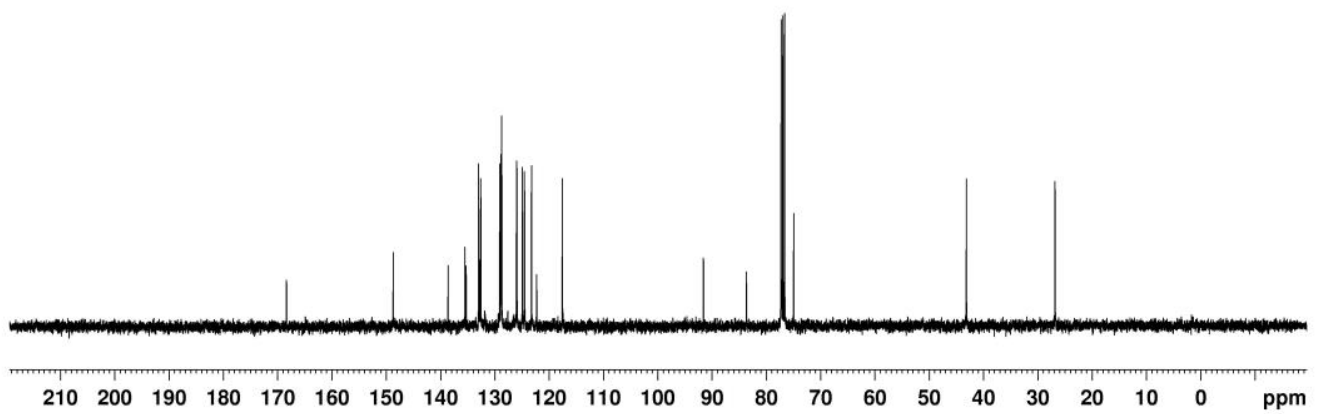




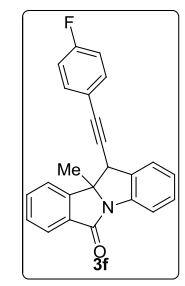

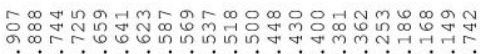

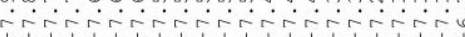
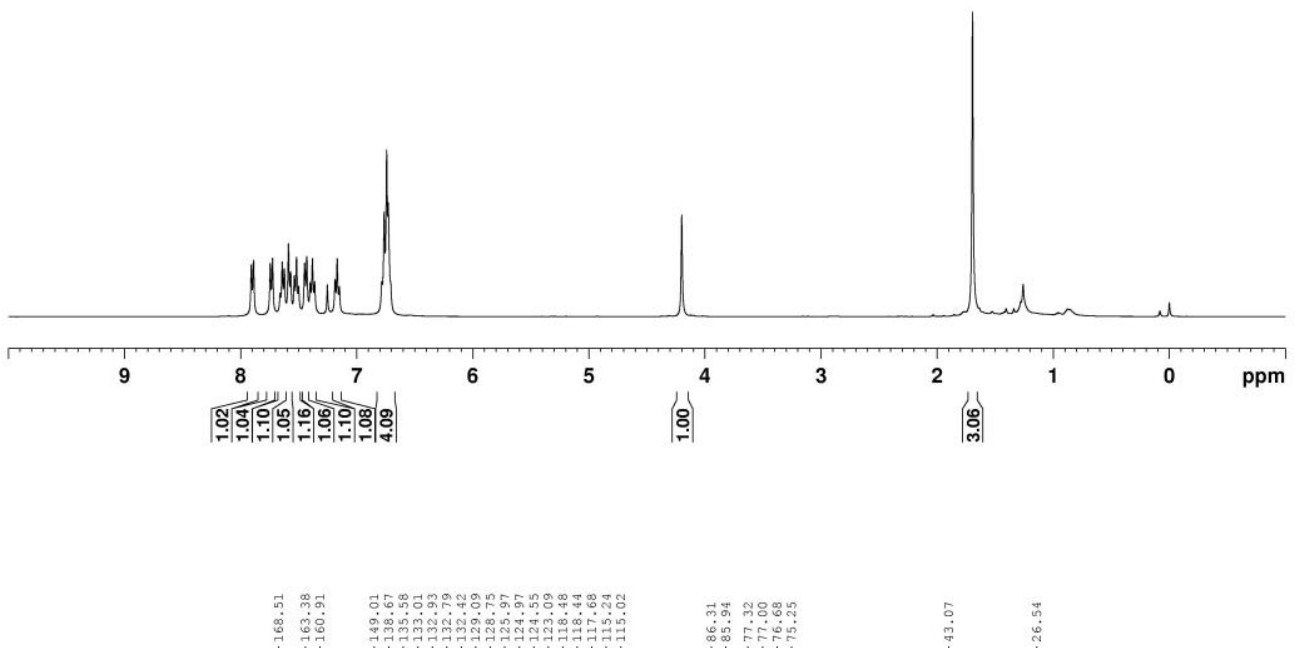

||

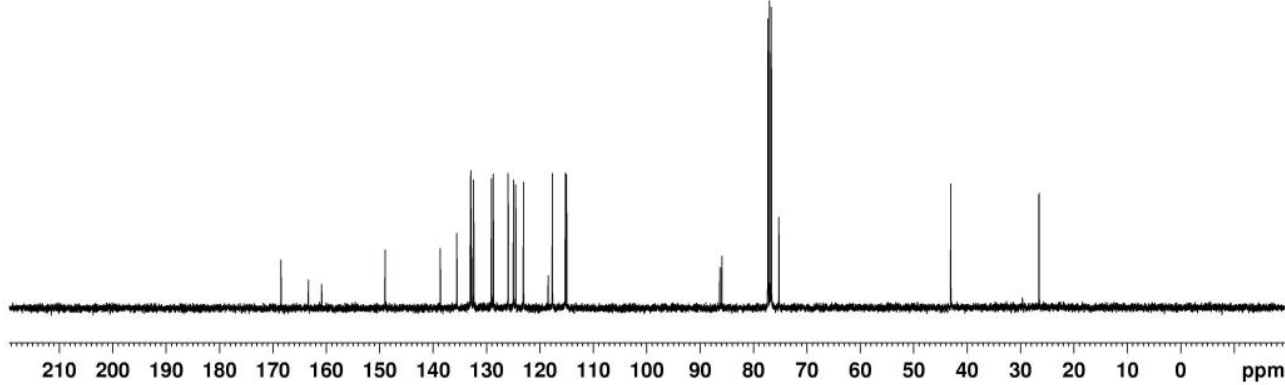



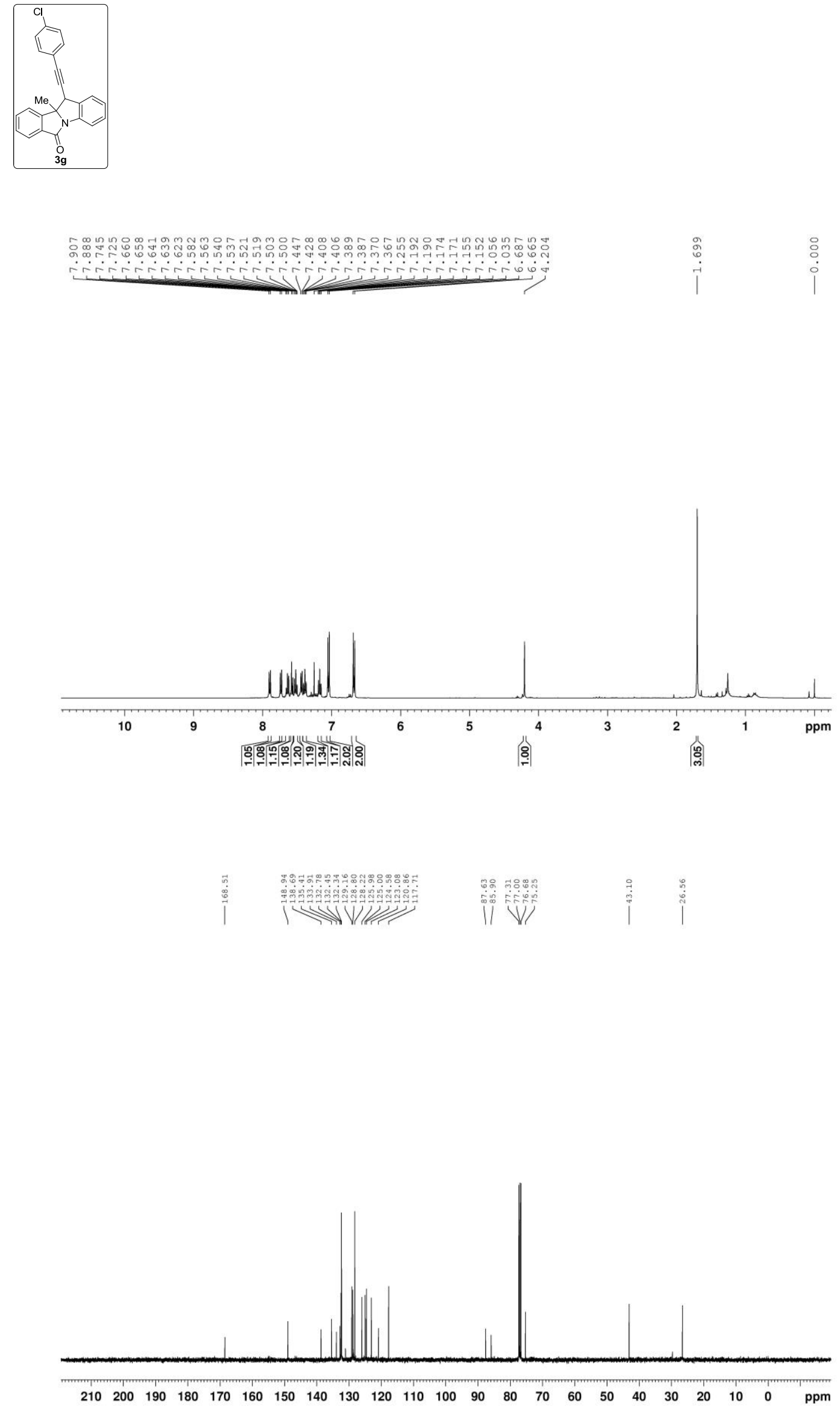

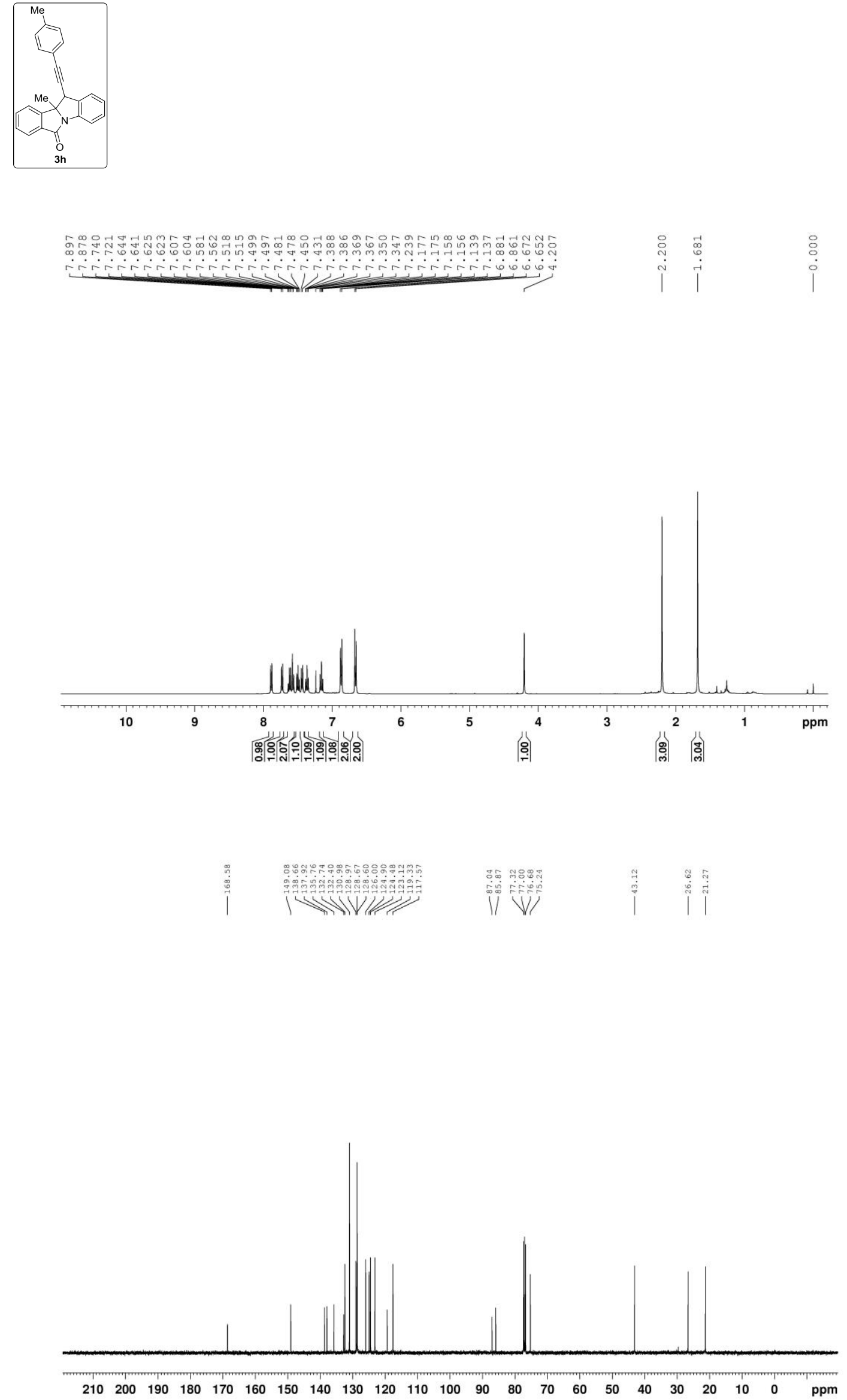


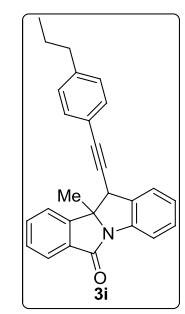

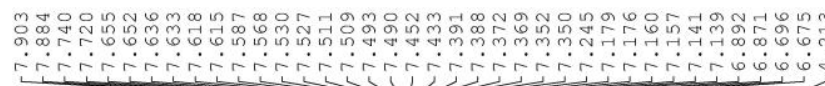

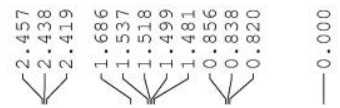

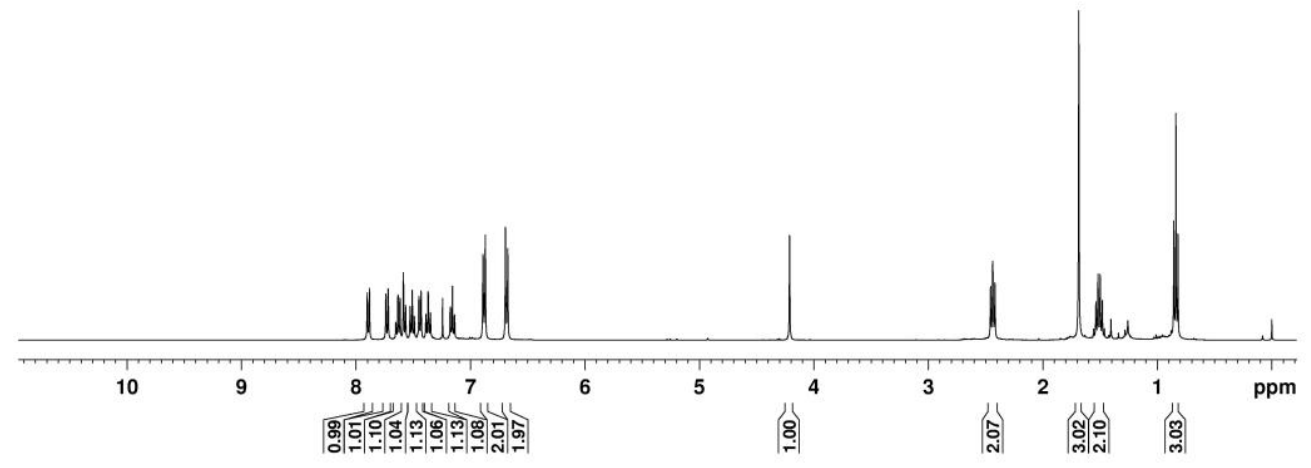

|

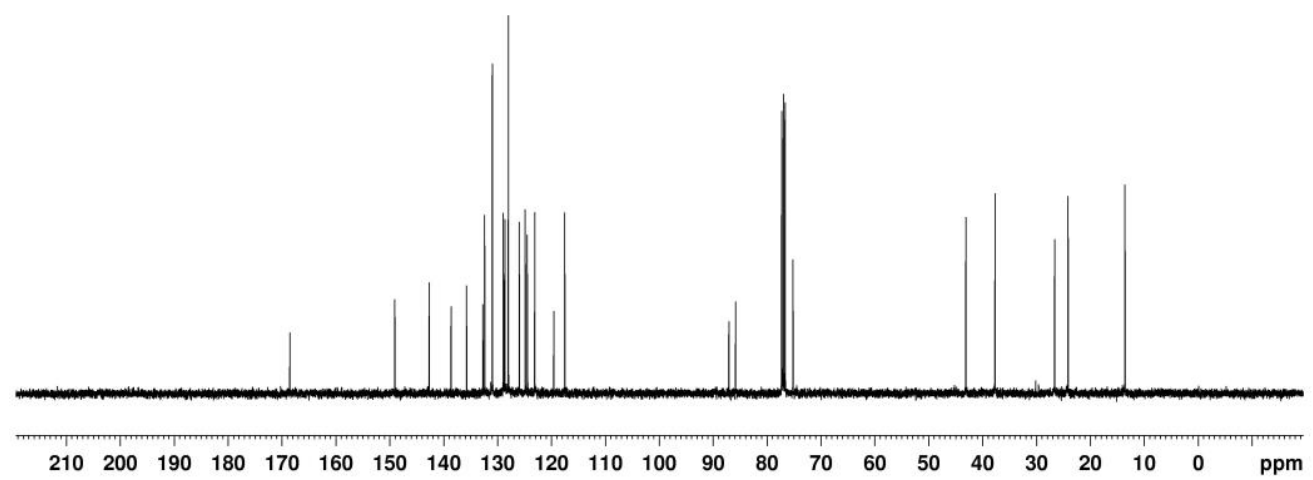




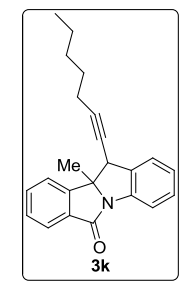

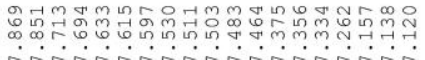
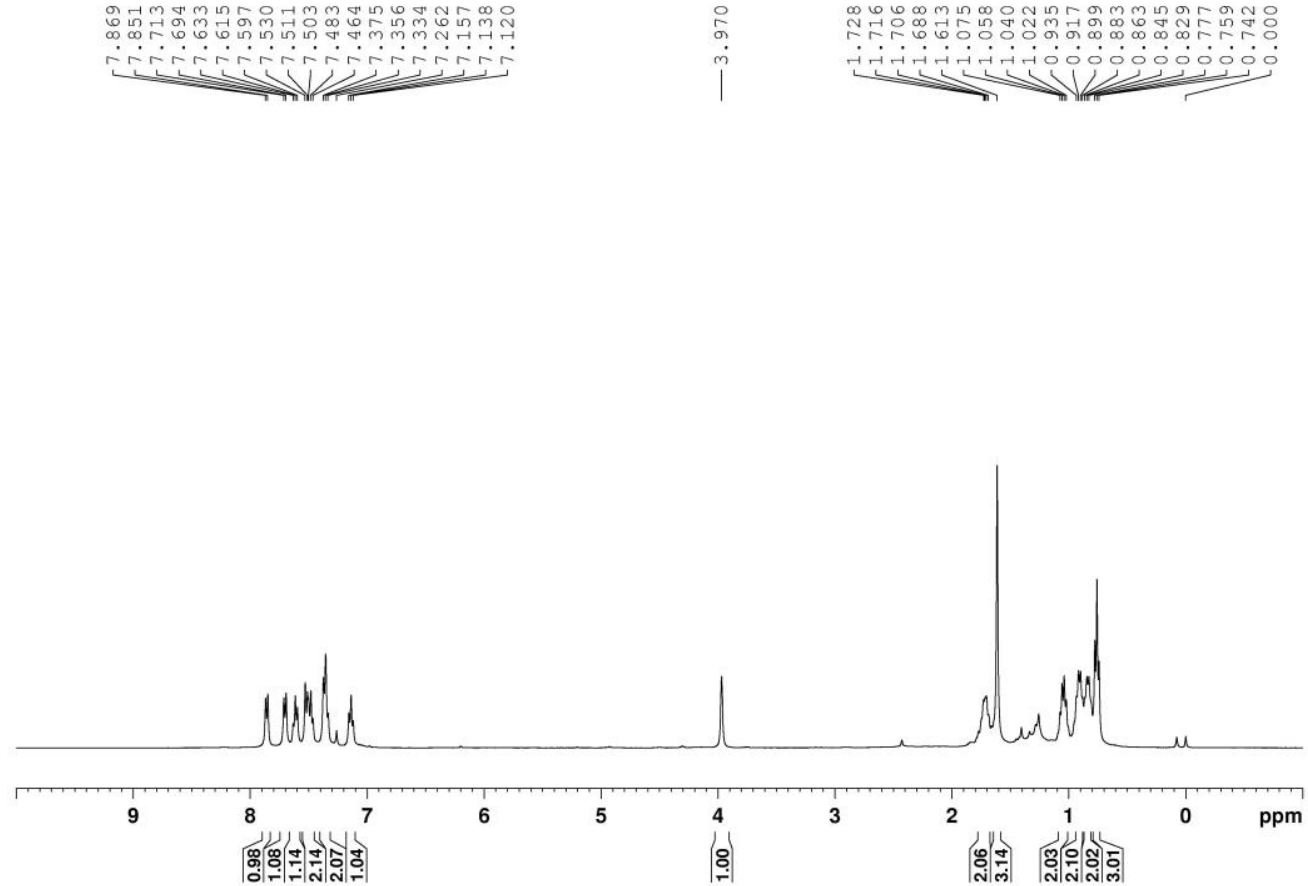

|

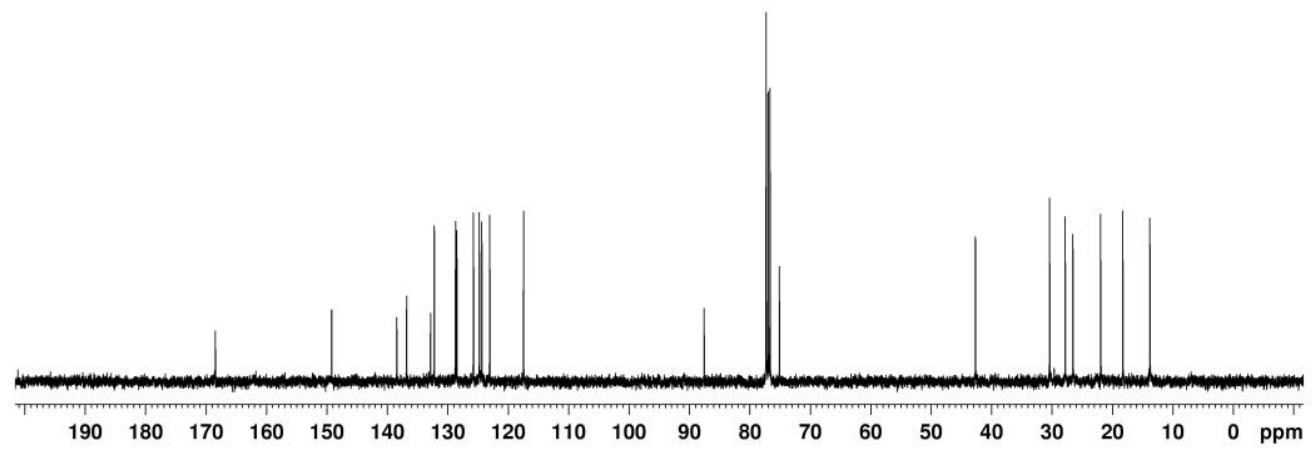




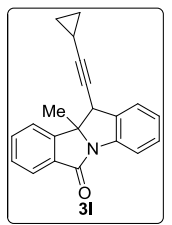

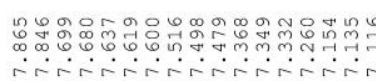
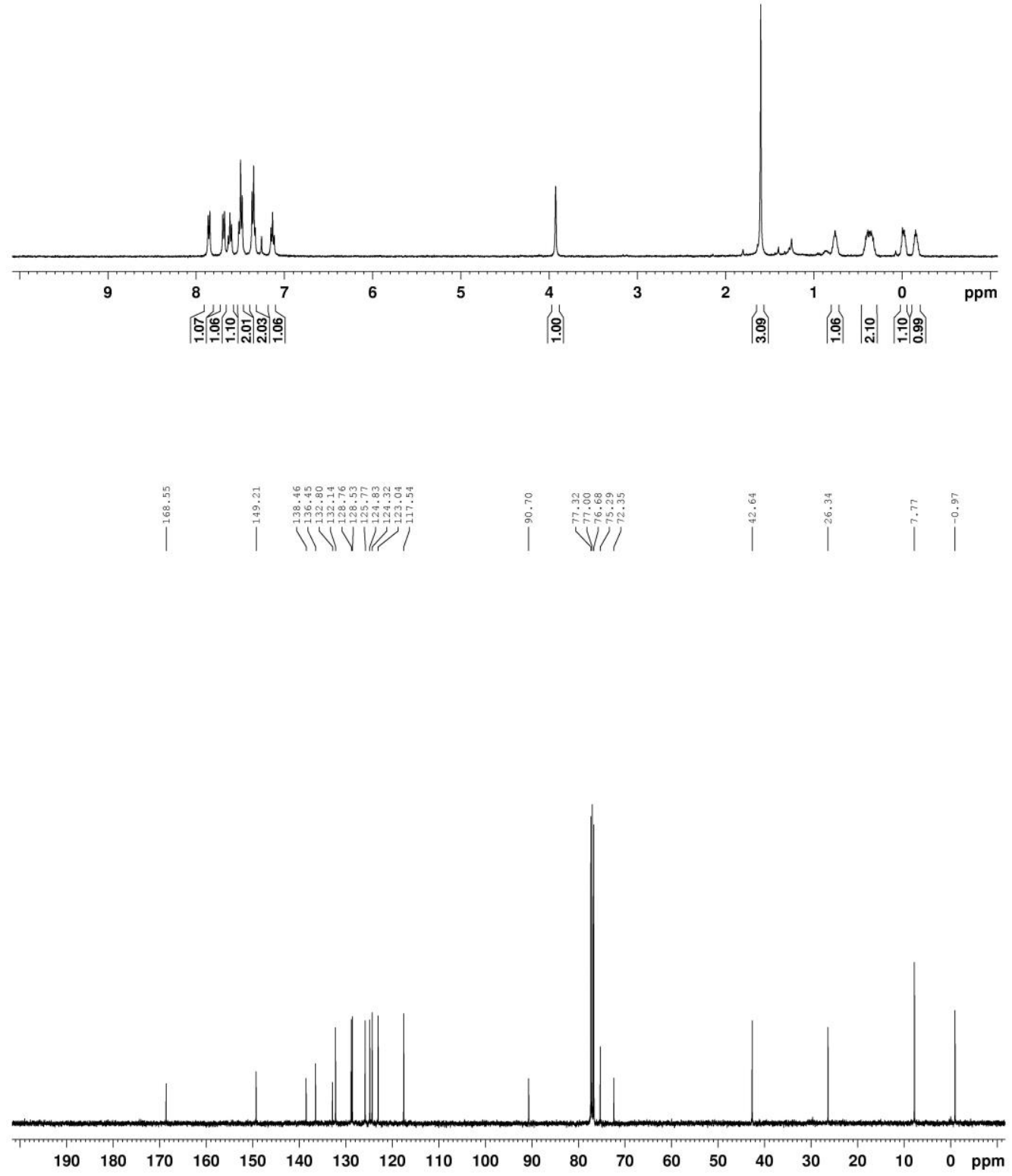


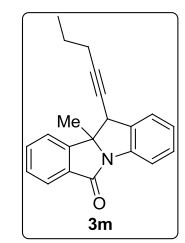

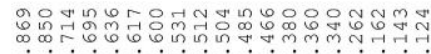

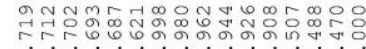

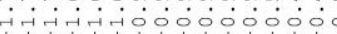

-

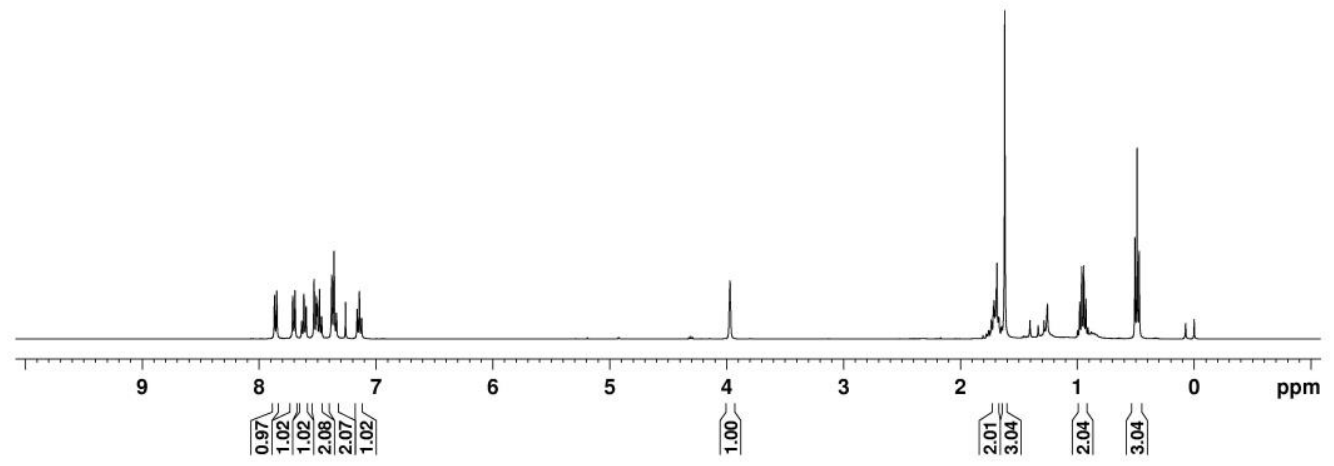

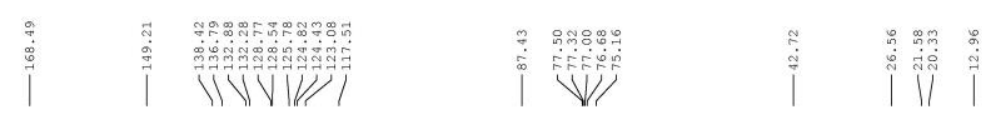

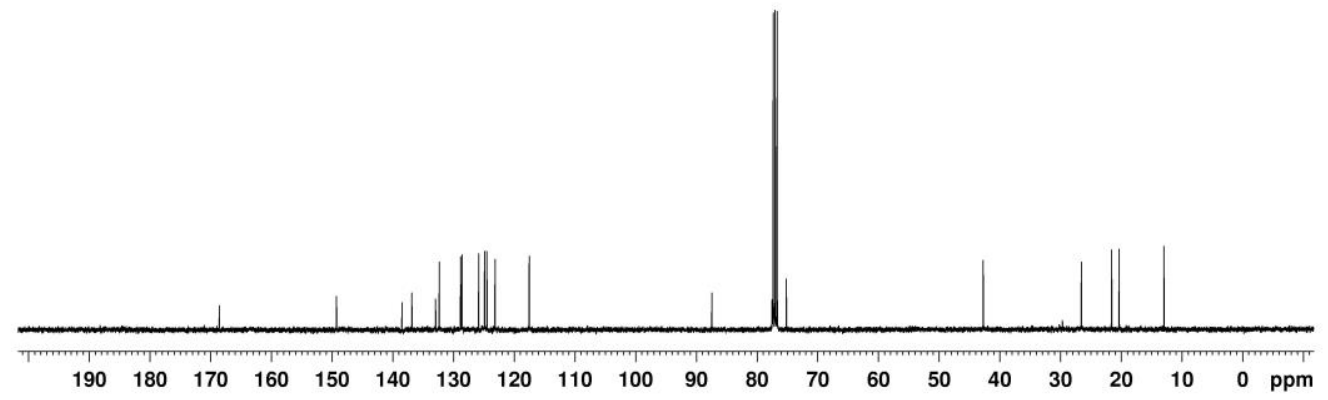




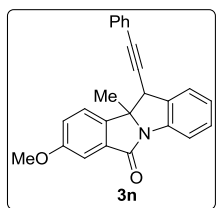

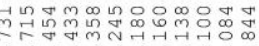

ann

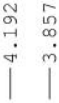
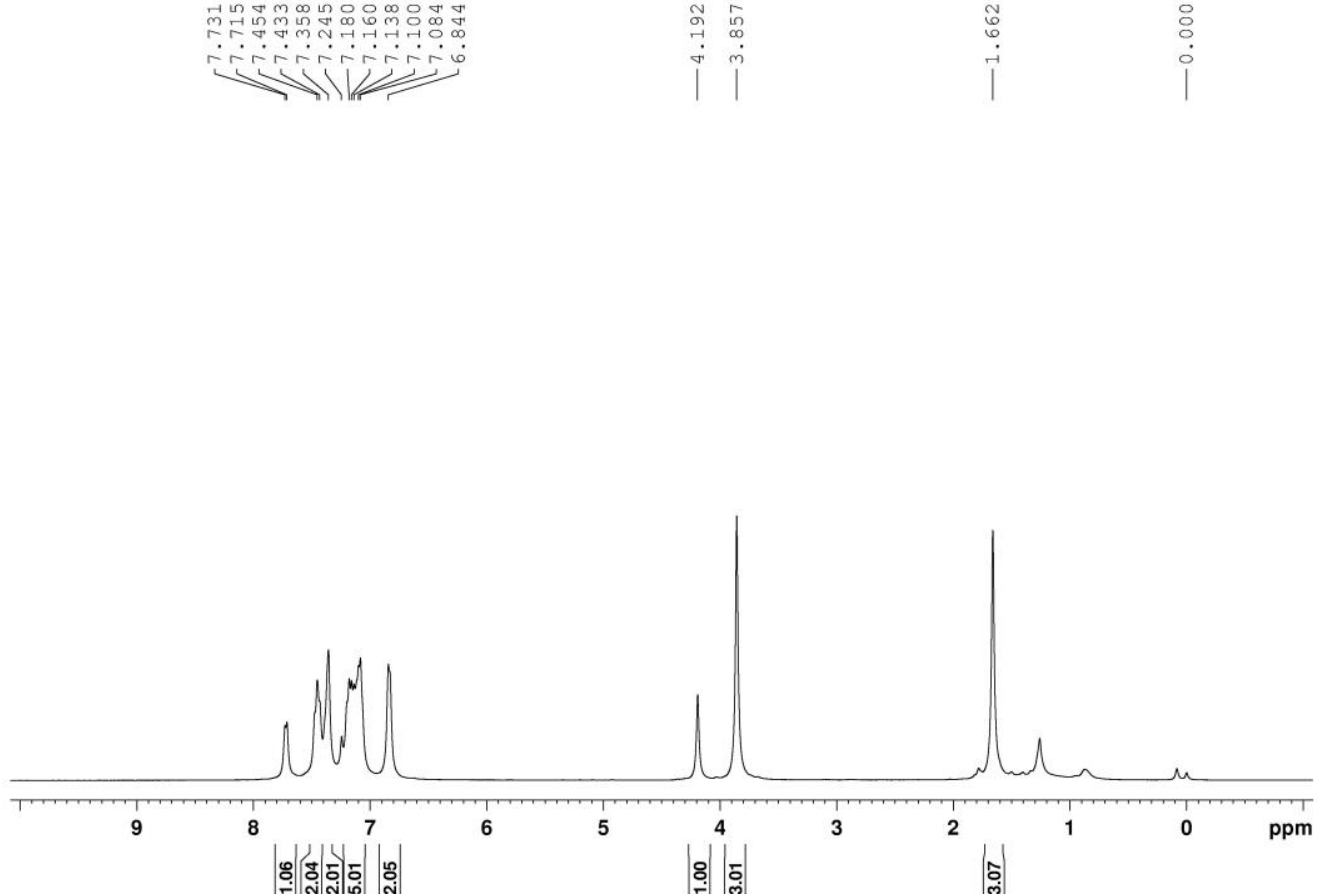

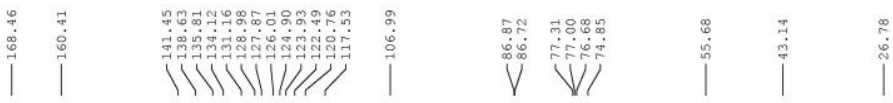

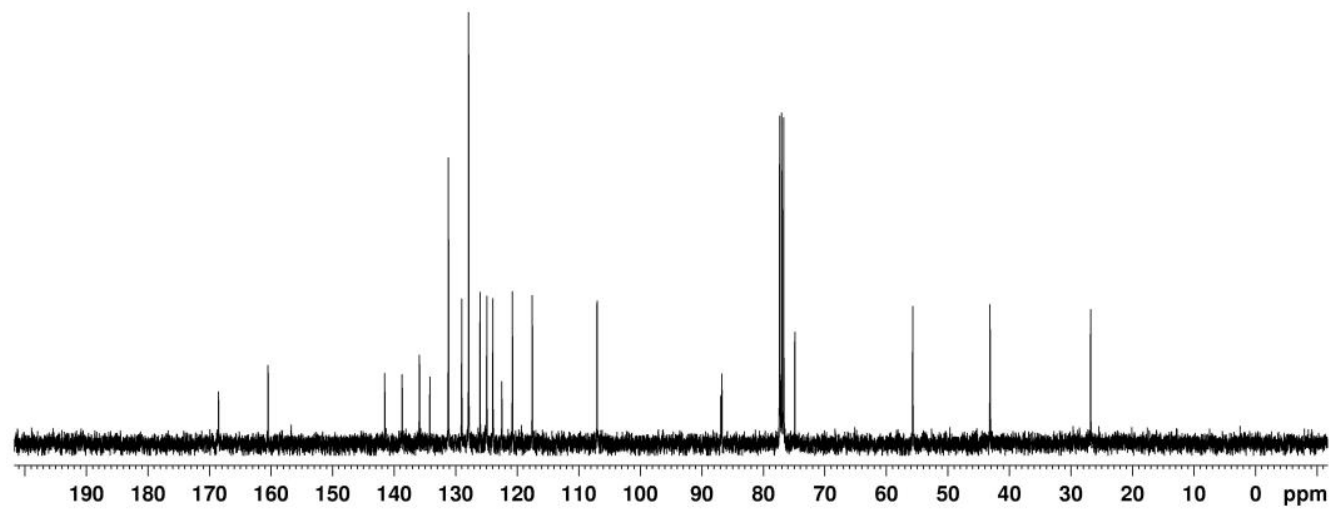




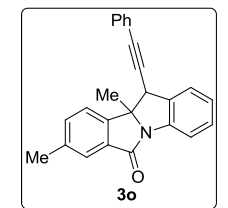

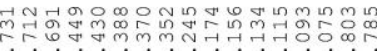

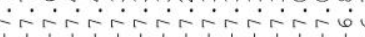

$\ldots+1$

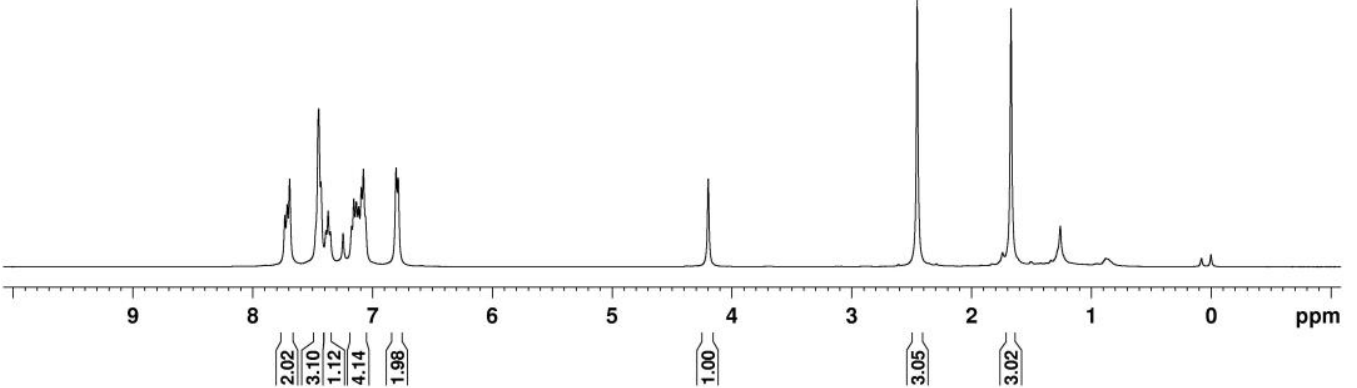

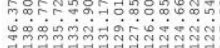

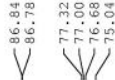

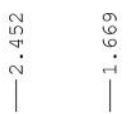

\section{웅}

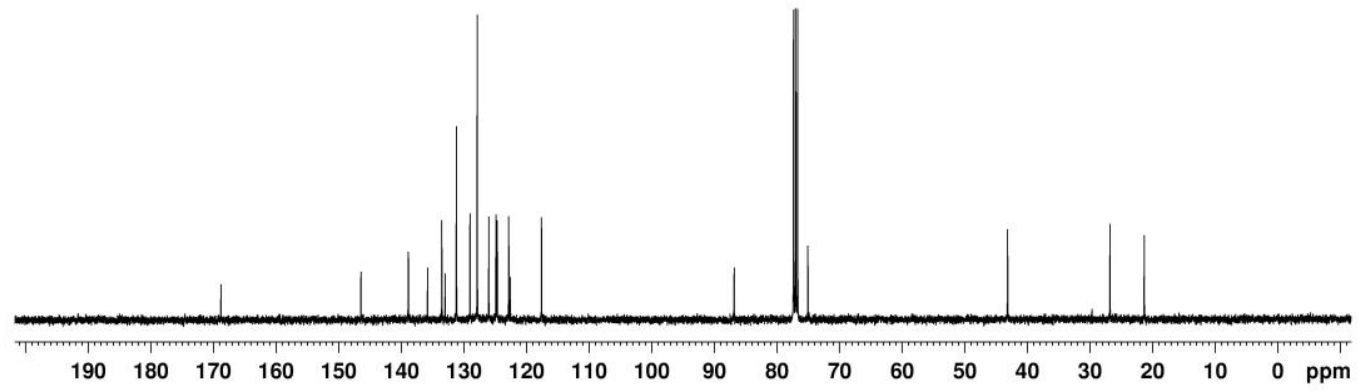



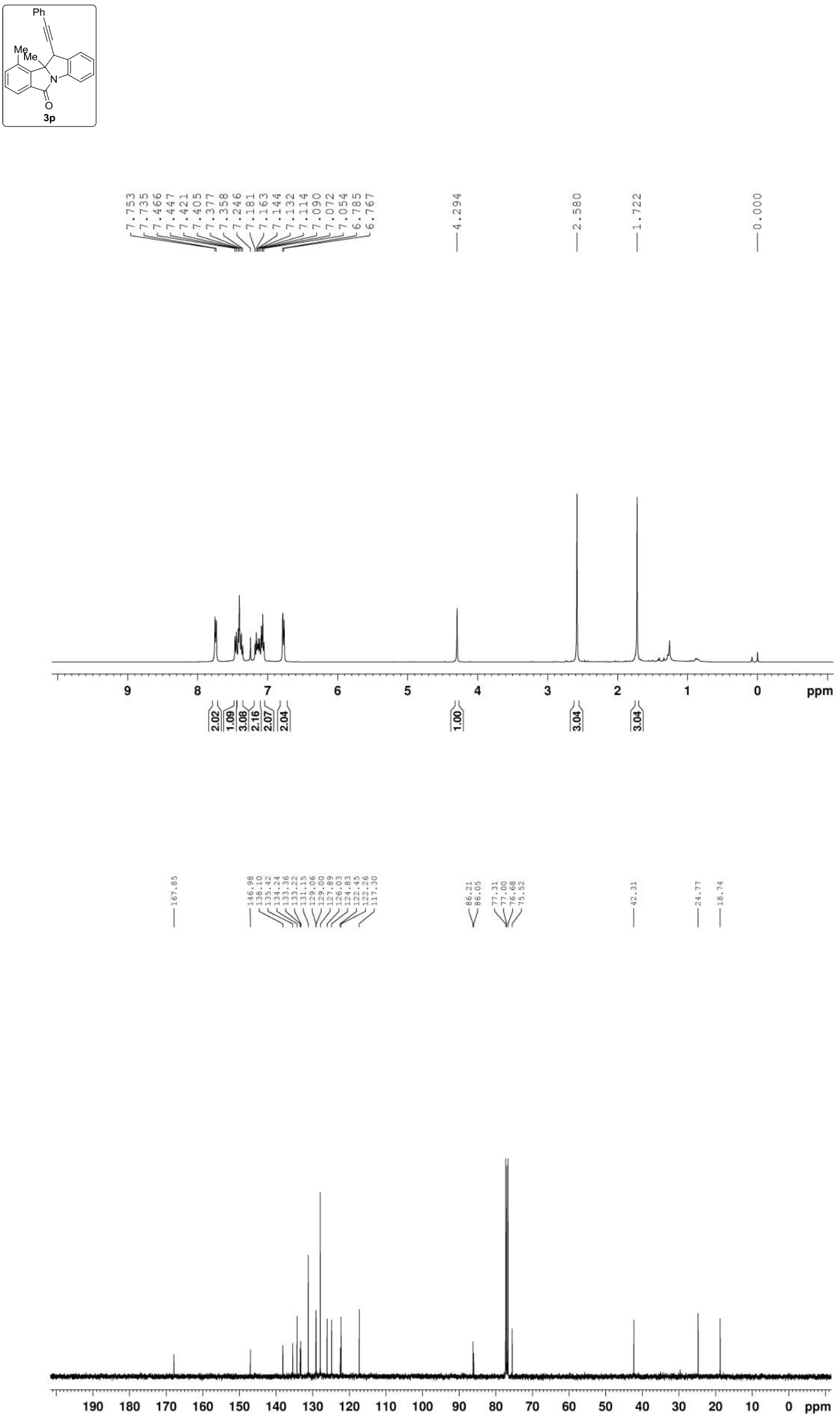

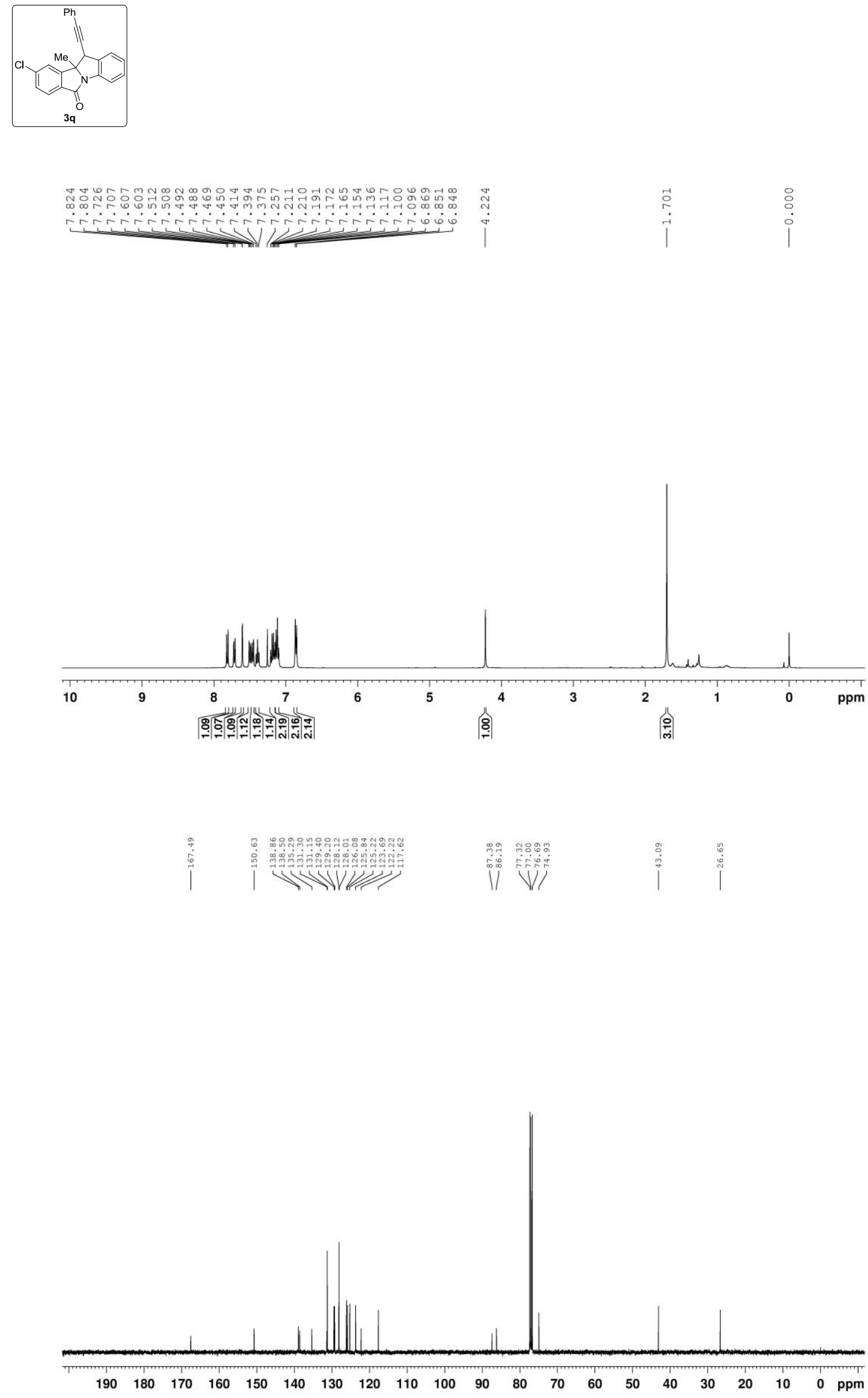


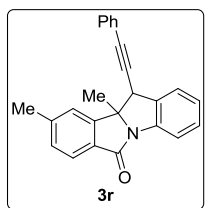

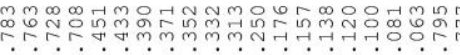

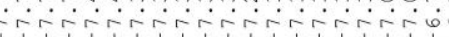

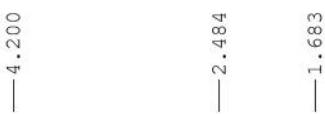

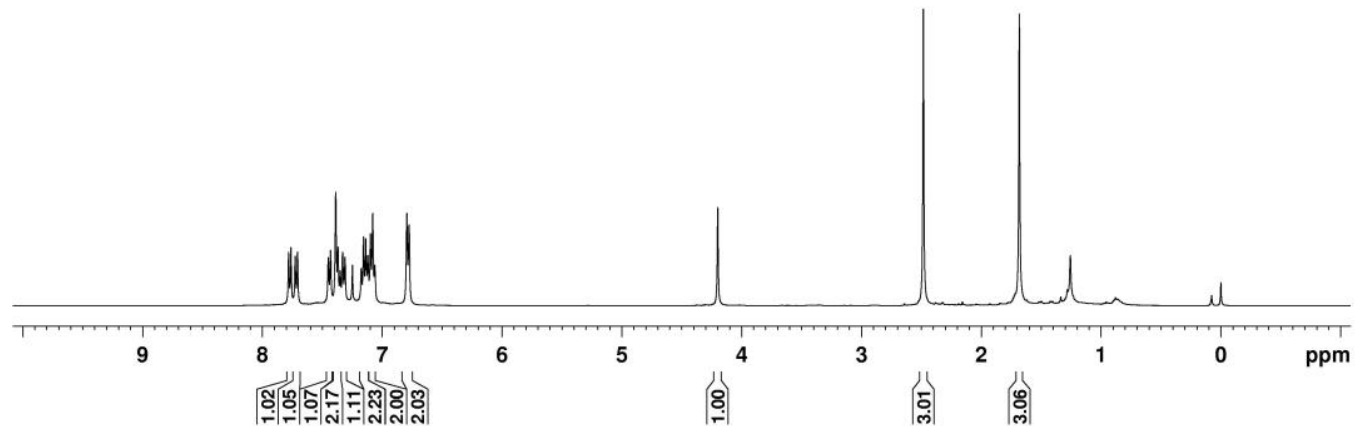

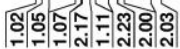

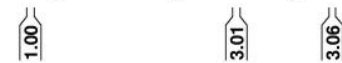

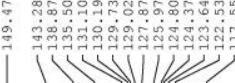

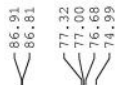

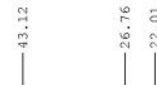

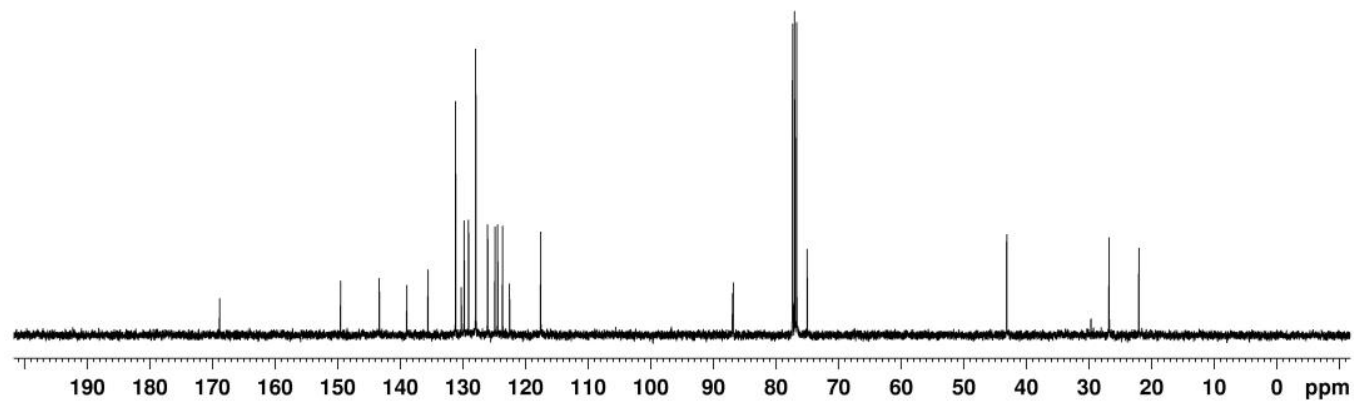



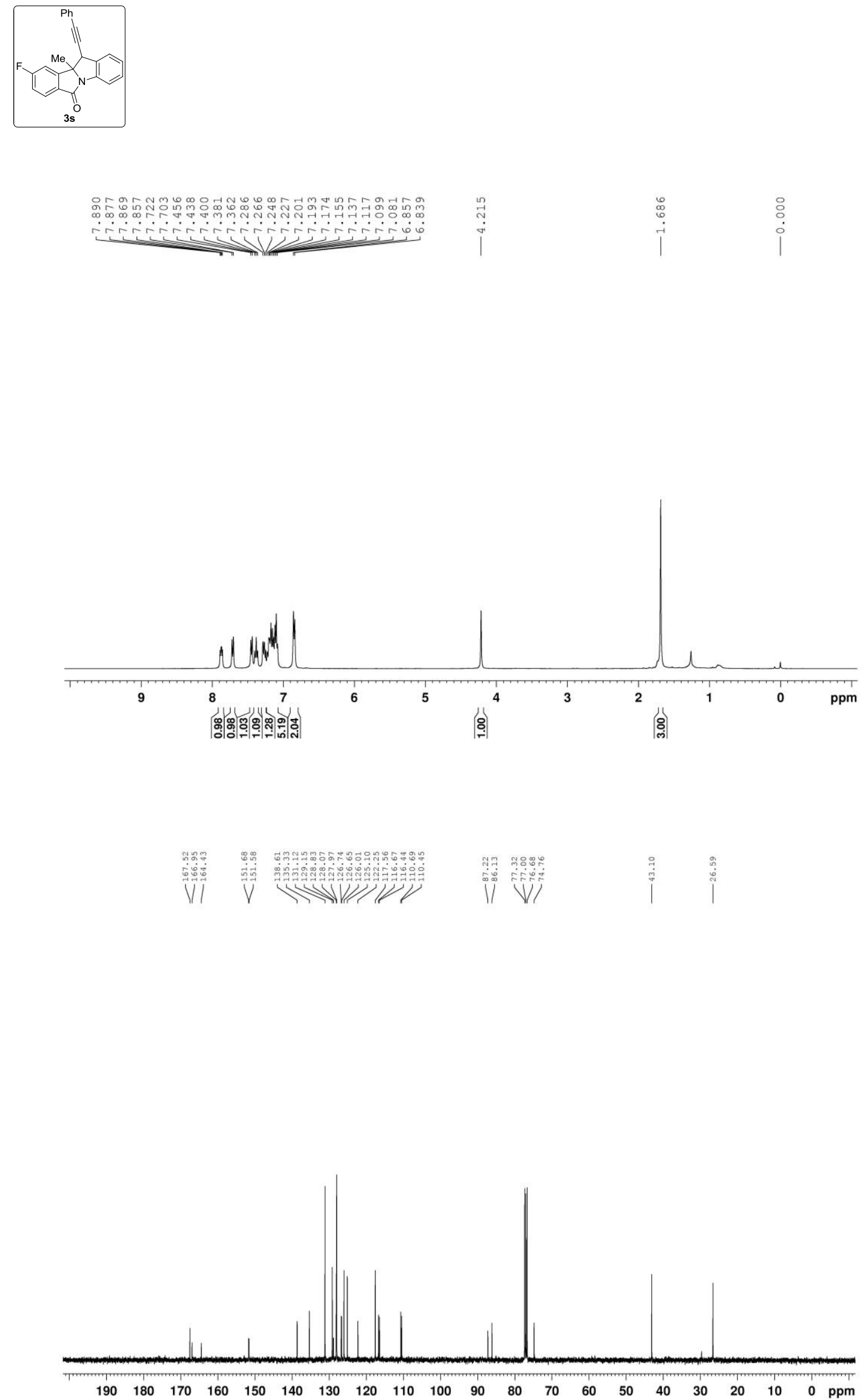


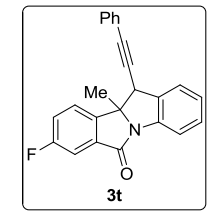

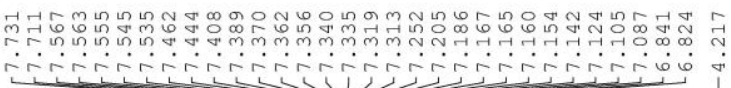
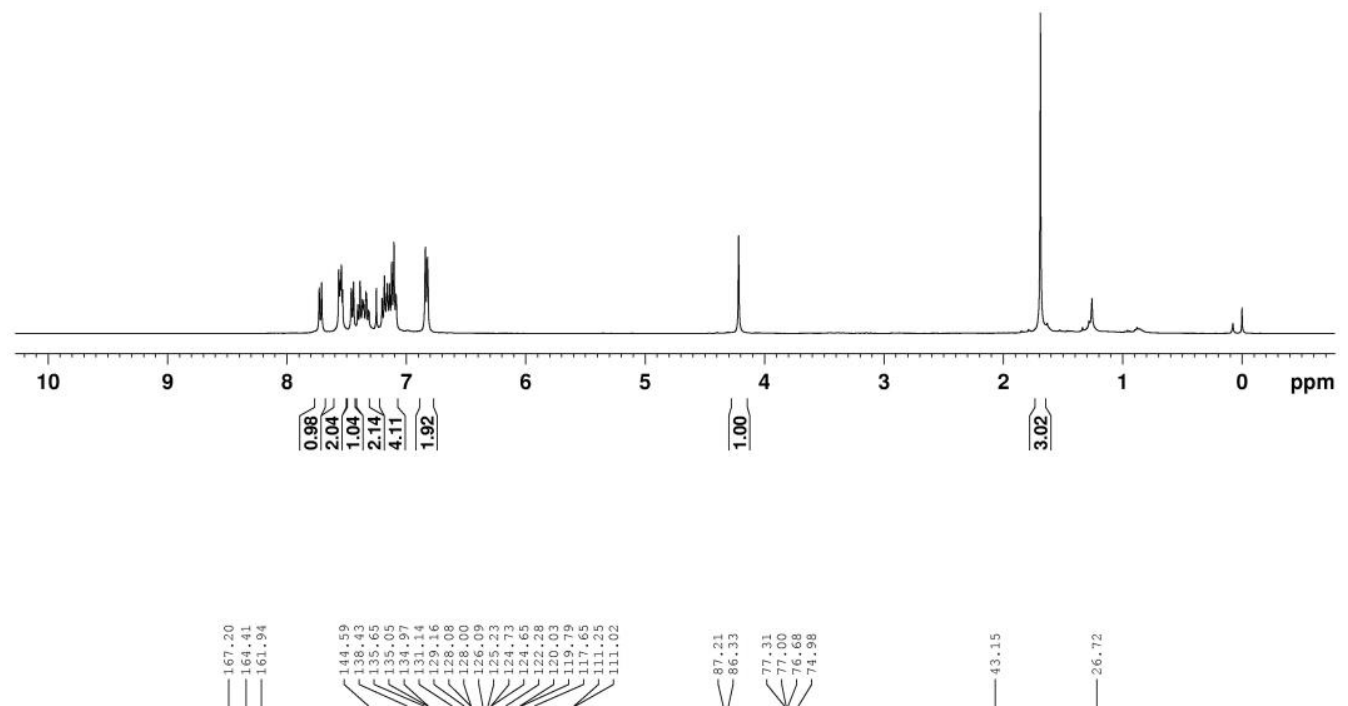

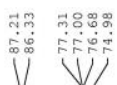

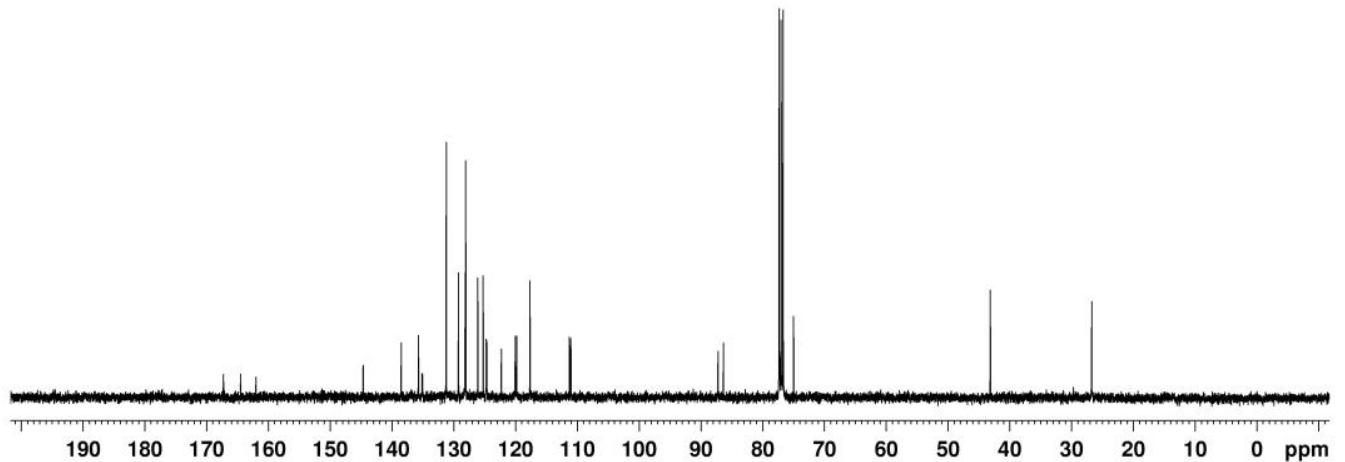




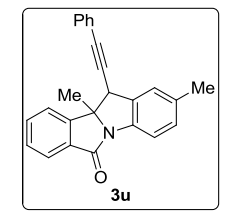

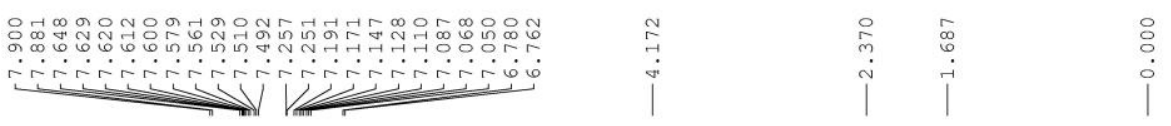
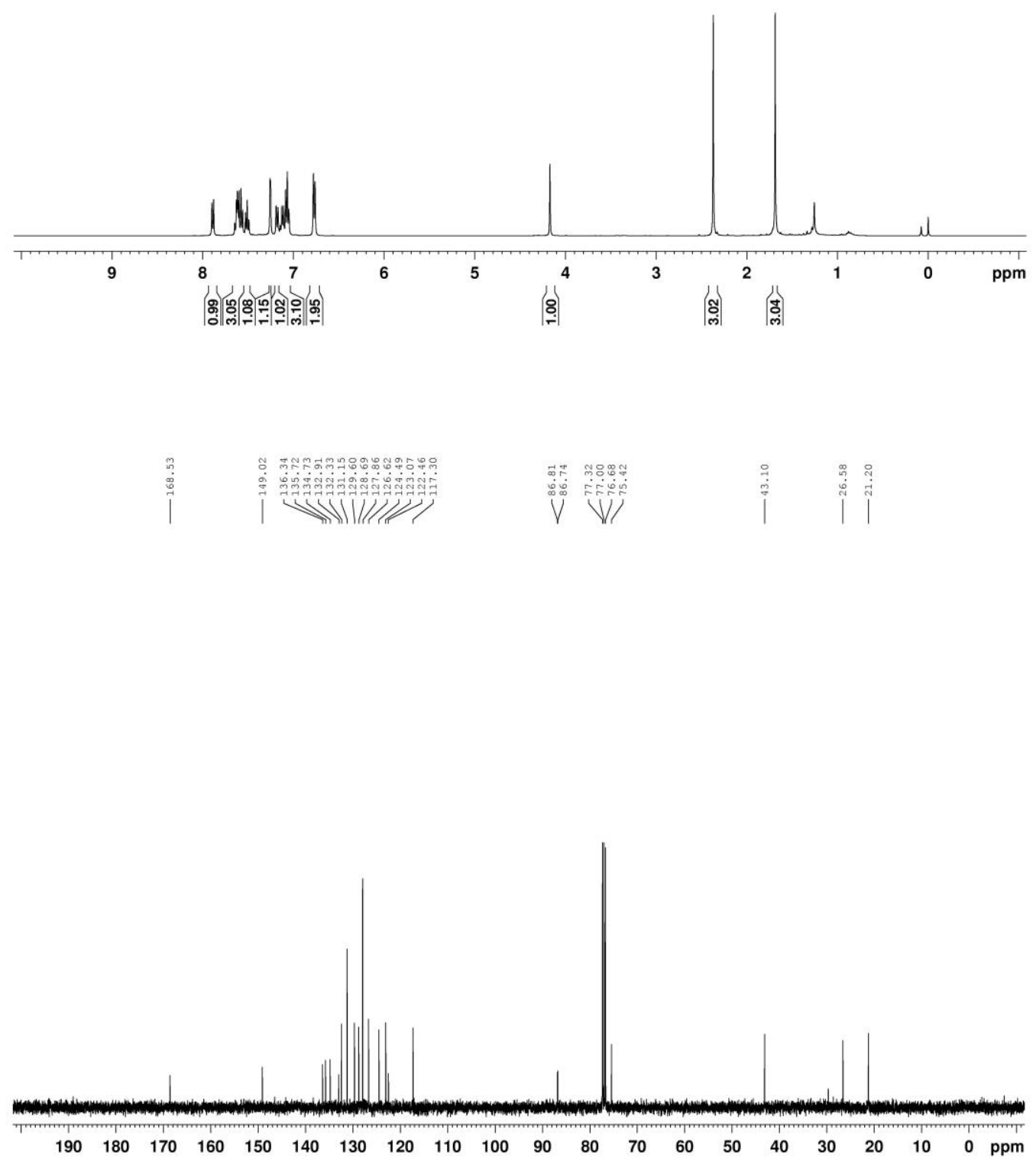


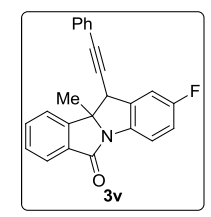

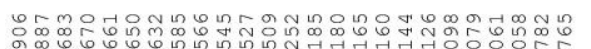

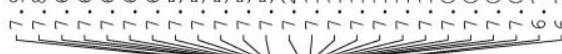
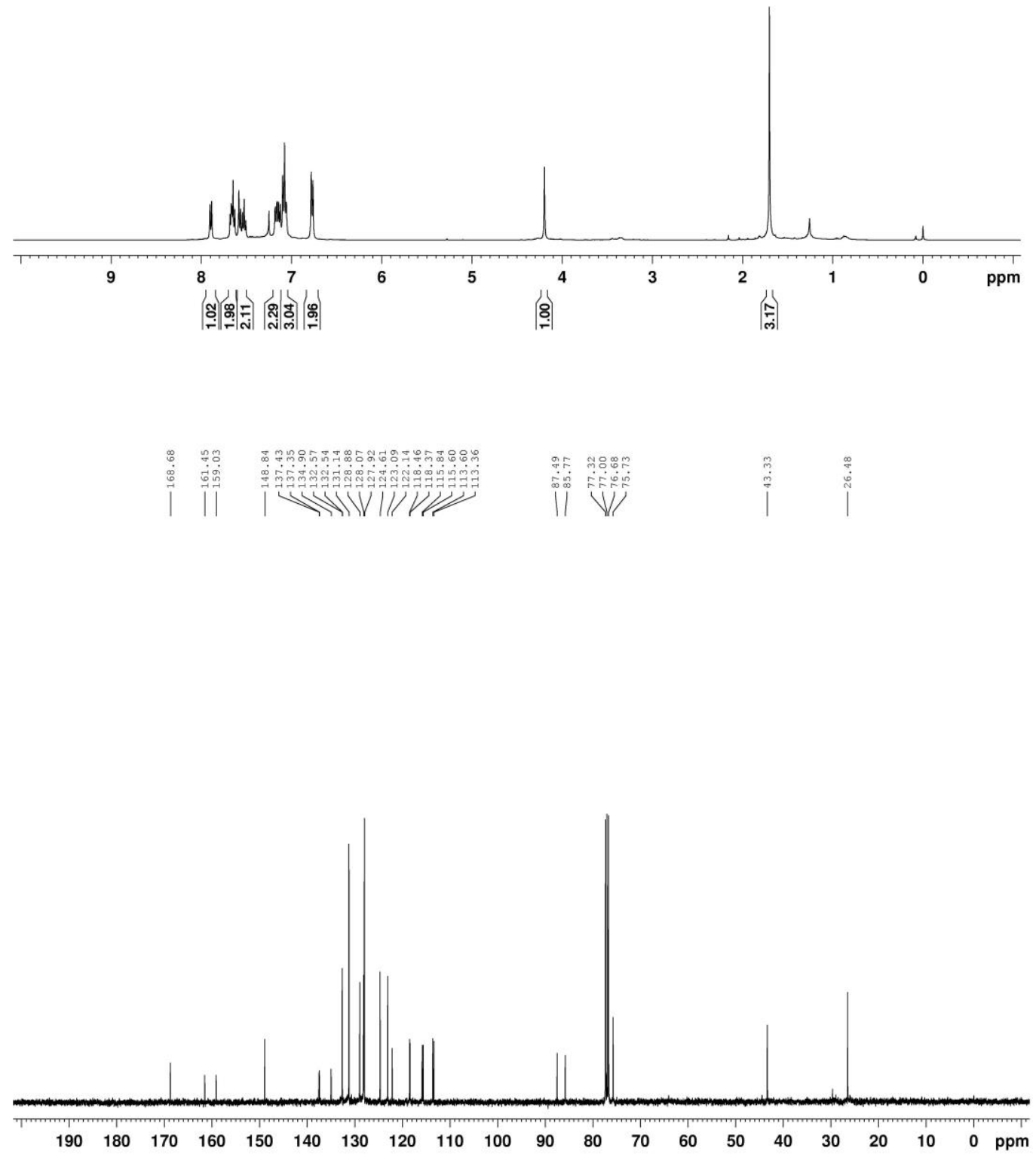


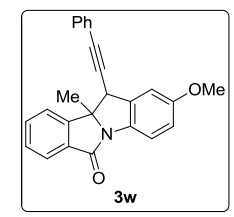

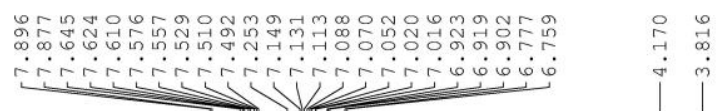
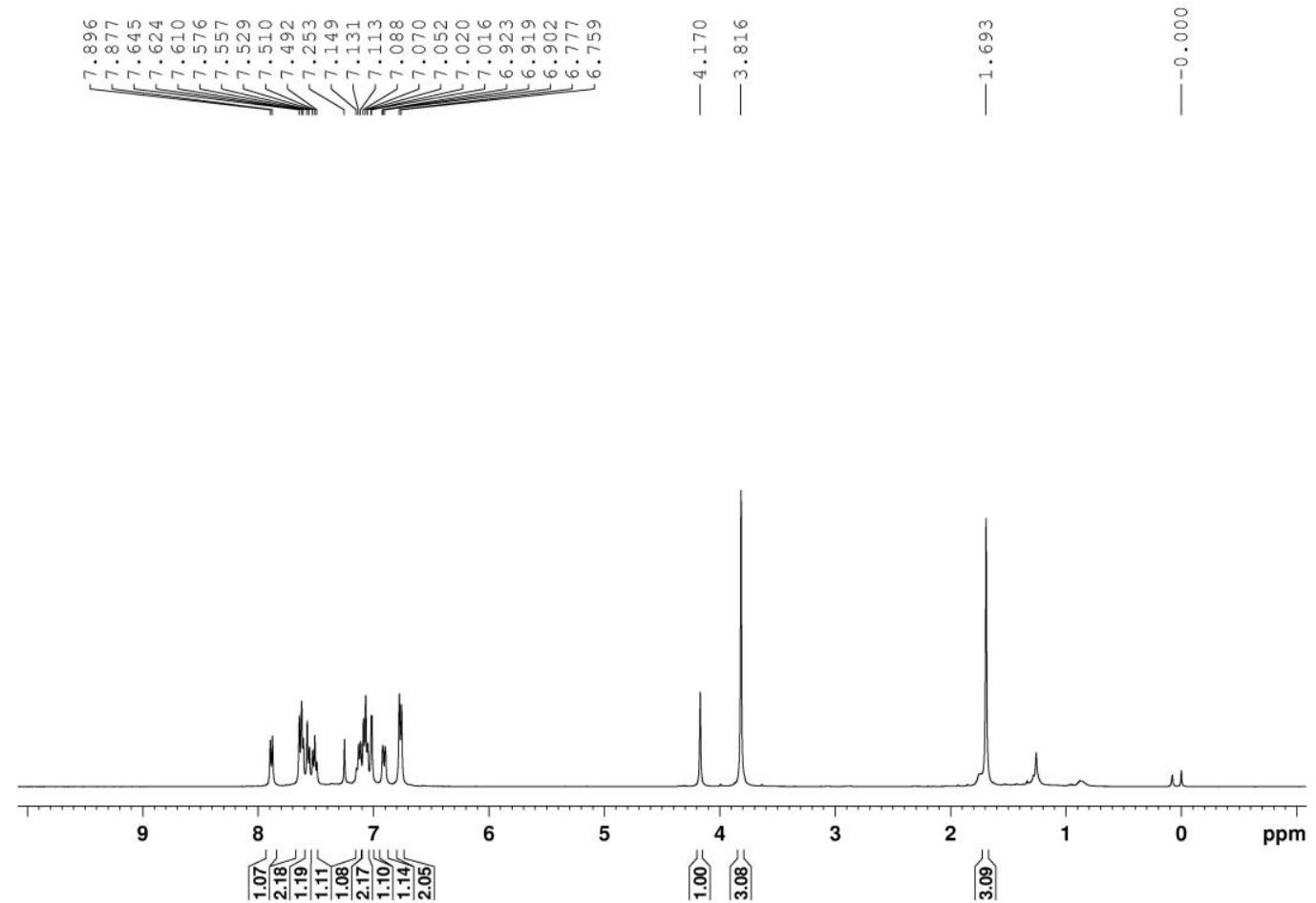

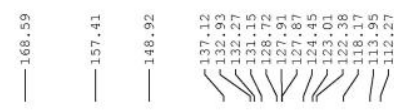

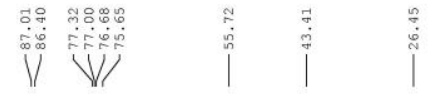

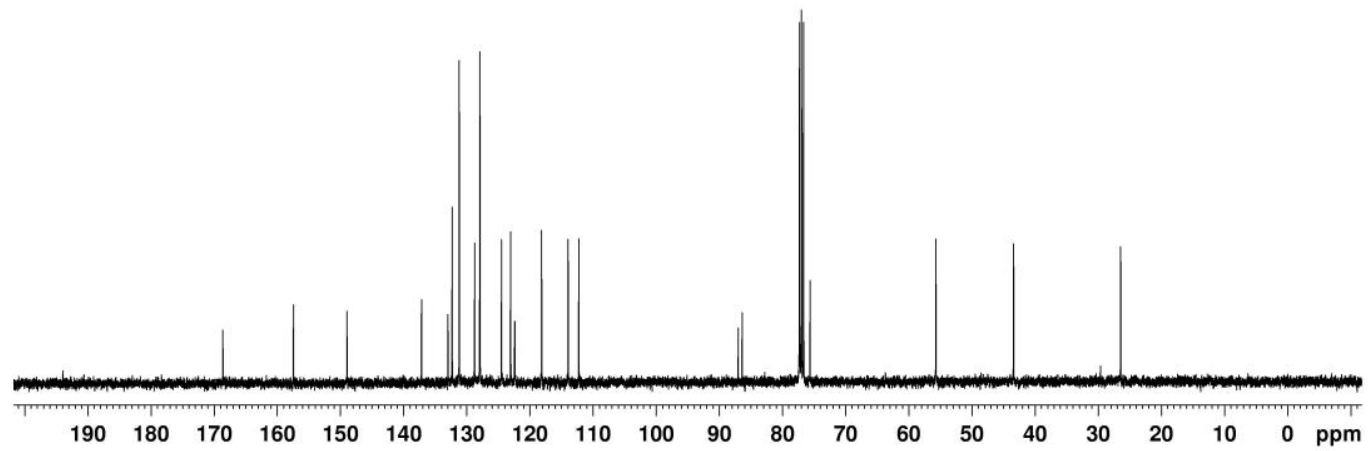



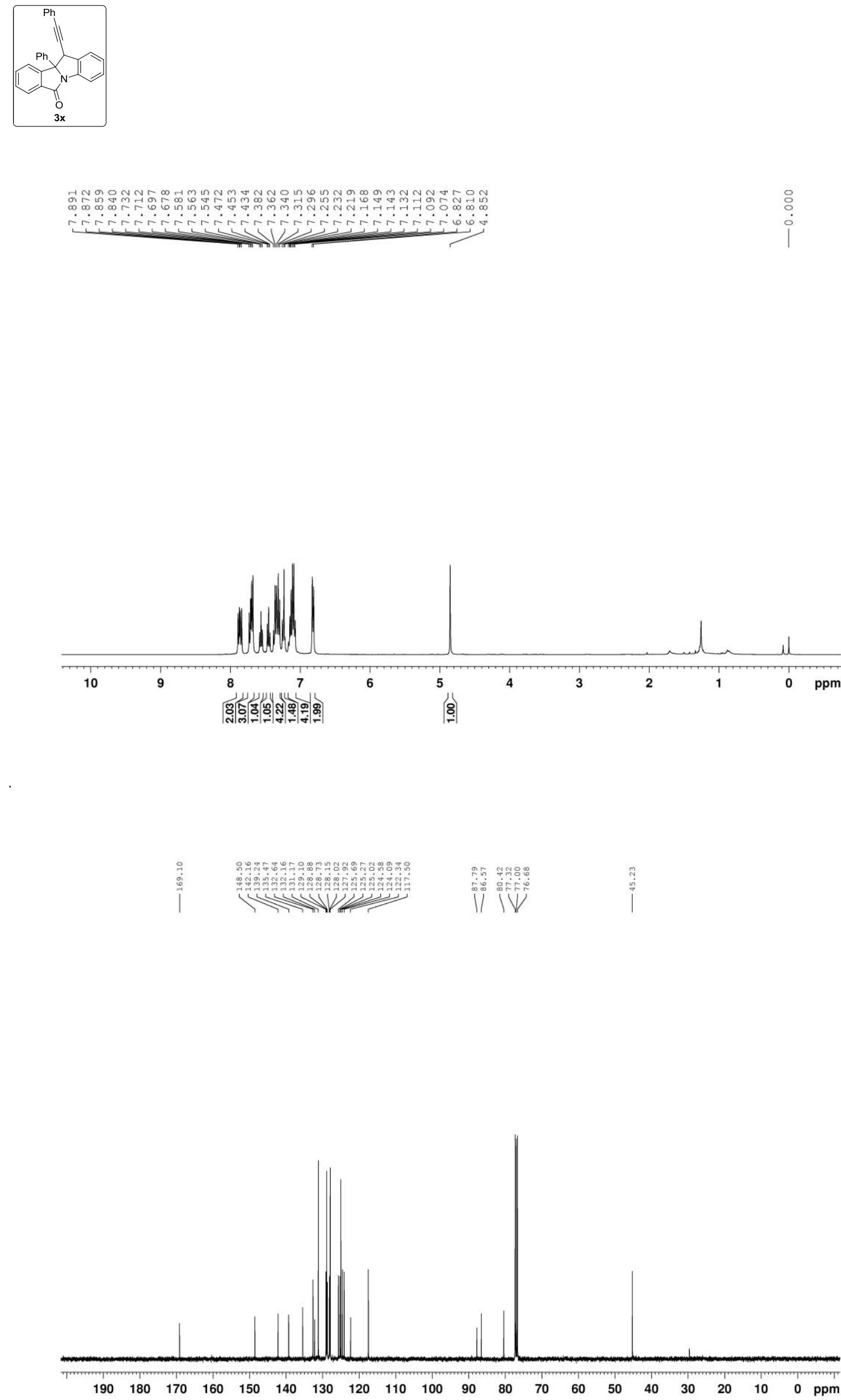


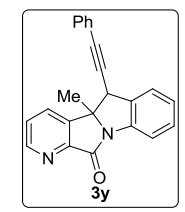

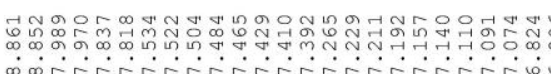

$\underbrace{\infty}_{0}$

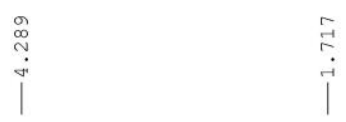

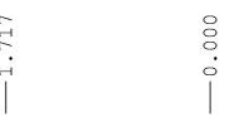
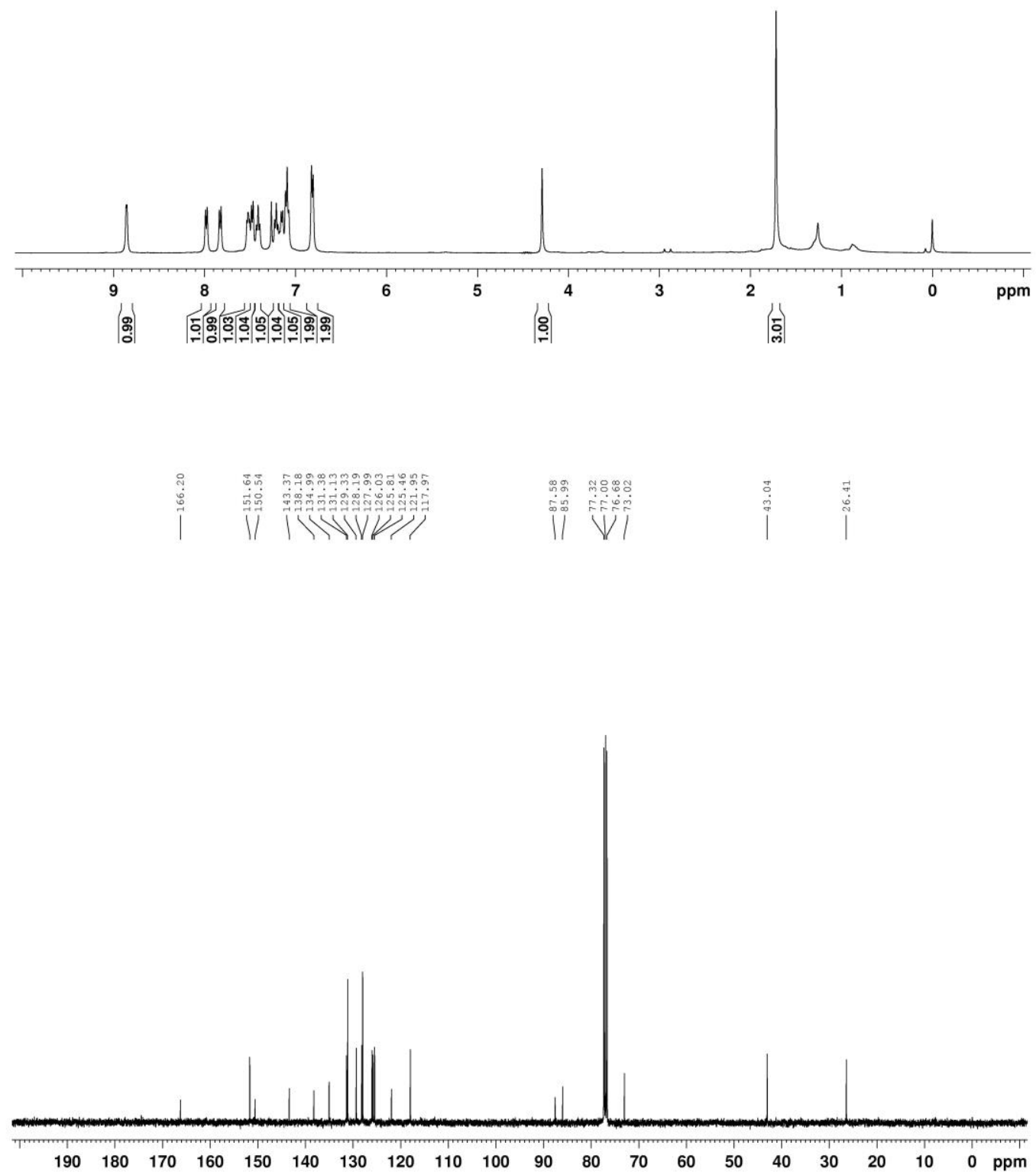


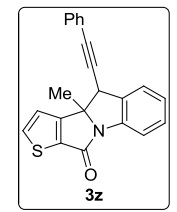

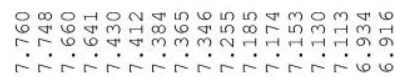

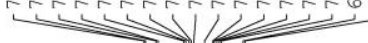
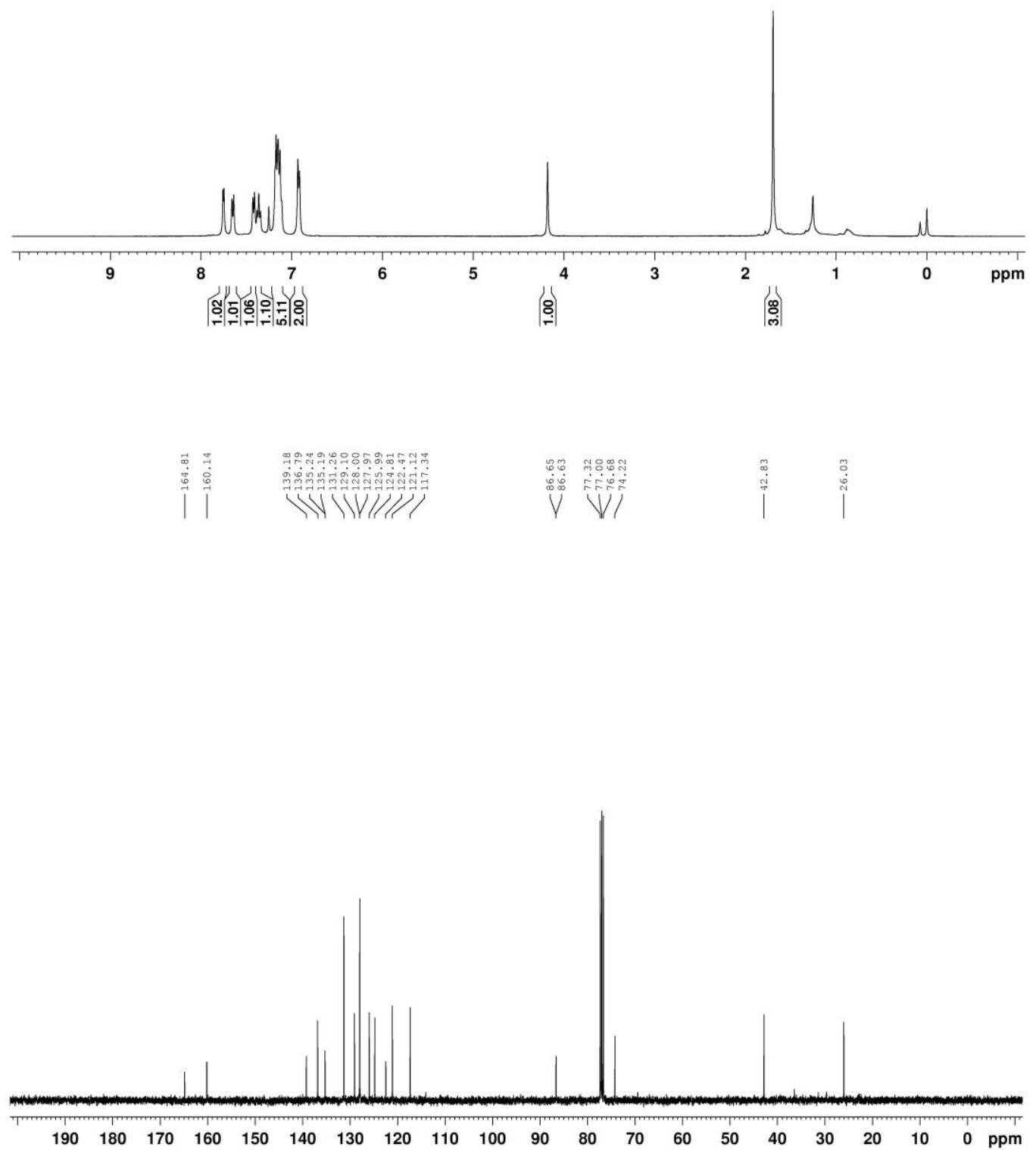


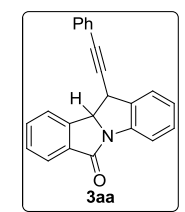

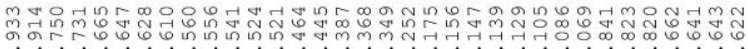

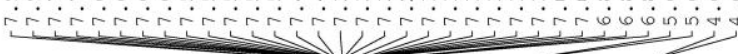
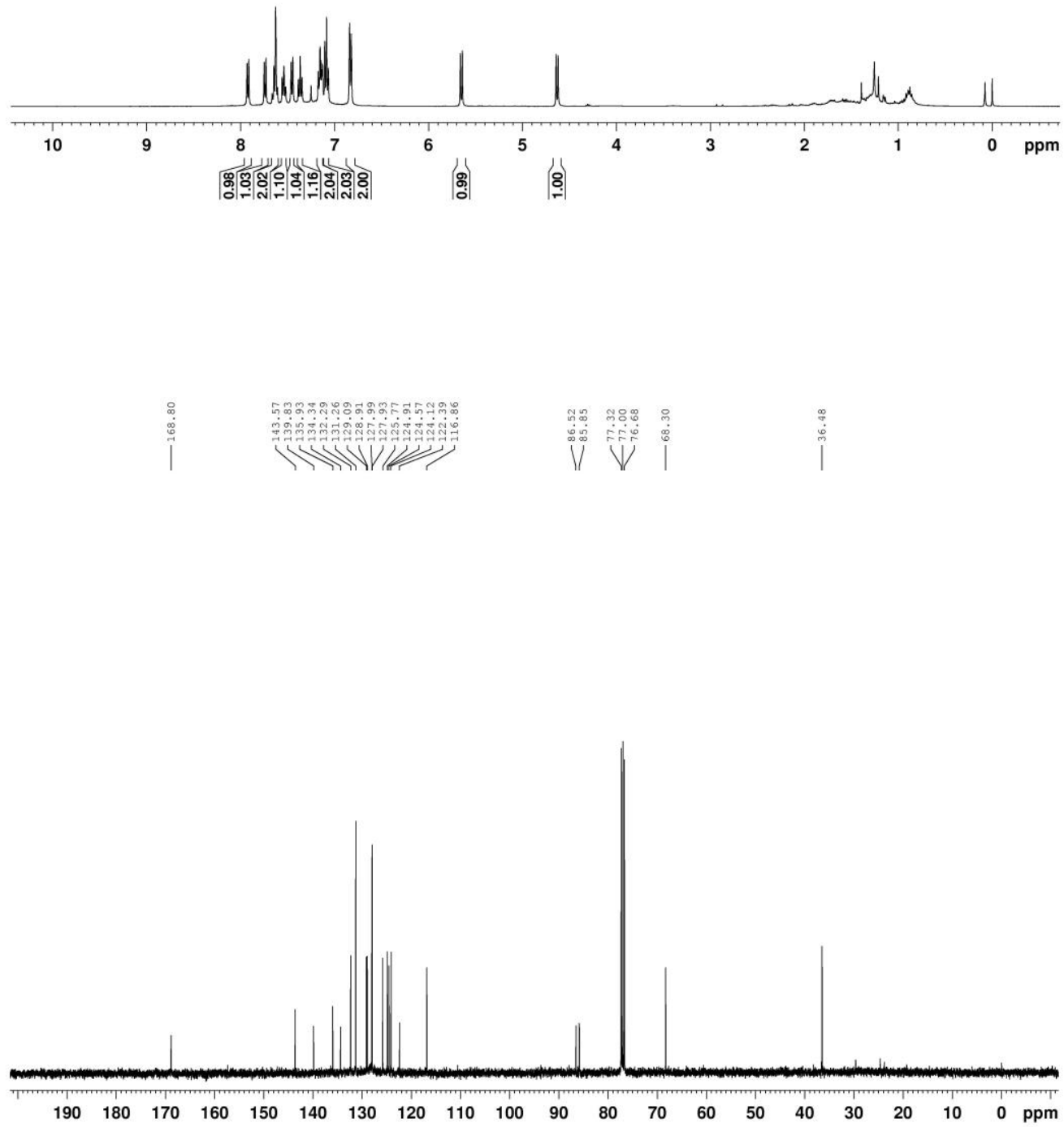